07

\title{
Квантовые флуктуации в лазерном солитоне
}

\author{
(С) Т.Ю. Голубева ${ }^{1}$, Ю.М. Голубев ${ }^{1}$, С.В. Федоров ${ }^{2}$, Л.А. Нестеров $^{2}$, Н.Н. Розанов ${ }^{2,3,4}$ \\ ${ }^{1}$ Санкт-Петербургский государственный университет, \\ 199034 Санкт-Петербург, Россия, \\ ${ }^{2}$ Физико-технический институт им. А.Ф. Иофрфе, \\ 194021 Санкт-Петербург, Россия \\ ${ }^{3} \mathrm{AO}$ „Государственный оптический институт им. С.И.Вавилова“, \\ 199034 Санкт-Петербург, Россия \\ ${ }^{4}$ Университет ИТМО, \\ 197101 Санкт-Петербург, Россия \\ e-mail: tania.golubeva@gmail.com, yuri.golubev@gmail.ru
}

Поступила в редакцию 11.12.2019 г.

В окончательной редакции 11.12.2019 г.

Принята к публикации 20.12.2019 г.

В рамках последовательной квантовой электродинамики построено уравнение Гейзенберга-Ланжевена для пространственного лазерного солитона. Детально обсуждены канонические переменные для поля генерации и для материальной двухуровневой среды, состоящей из среды, формирующей лазерную генерацию, и среды, осуществляющей насыщенное поглощение. Предполагается, что лазерная генерация развивается во времени значительно медленнее, чем атомная среда. Это дает возможность применить адиабатическое приближение и построить замкнутое уравнение для амплитуды лазерного поля. При выводе уравнения особое внимание уделено определению ланжевеновских источников, которые играют решающую роль в формировании квантовых статистических особенностей солитонов. Для того чтобы обеспечить процедуру наблюдения квантового сжатия солитона, предусмотрена синхронизация лазерной генерации внешним слабым электромагнитным воздействием.

Ключевые слова: лазерный солитон, квантовое сжатие, уравнение Гейзенберга-Ланжевена.

DOI: 10.21883/OS.2020.04.49203.334-19

\section{1. Введение}

Диссипативные оптические солитоны [1] представляют значительный интерес, поскольку реализующийся в них динамический баланс притока и оттока энергии обеспечивает их повышенную устойчивость по сравнению с более изученными консервативными солитонами [2]. Предельные возможности информационных приложений диссипативных солитонов диктуются квантовыми флуктуациями поля и среды, в которой они распространяются. Для консервативных временных солитонов теоретически предсказаны и экспериментально реализованы сжатые состояния отвечающего солитонам излучения с уровнем шумов, меньшим, чем уровень шума в обычном когерентном состоянии [3-5]. Аналогичные исследования квантовых флуктуаций пространственных консервативных солитонов выполнены в [6-8].

Для пространственных диссипативных оптических солитонов квантовые эффекты исследованы не столь детально. Результаты по генерации сжатого излучения таких солитонов в схеме параметрического генератора суммированы в монографии [9]. В [10-12] (см. также [1]) для пространственных солитонов в широкоапертурном пассивном нелинейном интерферометре с когерентным поддерживающим излучением выведено квантовое уравнение Ланжевена, решение которого позволило найти статистическую дисперсию флуктуаций среднего положения и импульса солитона и условия реализации сжатых состояний.

Однако к настоящему времени отсутствуют исследования квантовых флуктуаций пространственных диссипативных солитонов в другой схеме - широкоапертурном лазере с насыщающимся поглощением. Такая схема, предложенная в [13] (анализ ее последующих исследований см. в [1]), выгодно отличается от схемы интерферометра высоким контрастом интенсивности в центре солитона по сравнению с его периферией. Развитие теории квантовых эффектов для таких лазерных солитонов и служит задачей настоящего сообщения. Для общности и в методических целях мы рассмотрим более универсальную схему широкоапертурного лазера с насыщающимся поглощением и внешним поддерживающим излучением. При нулевой интенсивности поддерживающего излучения эта схема сводится к собственно лазерной, а при замене материальных уравнений для среды - к схеме нелинейного интерферометра с поддерживающим излучением.

Работа организована следующим образом. Раздел 1 является вводным. В разд. 2 обсуждается принятая в работе физическая модель генерации лазерного солитона. В разд. 3 обсуждаются канонические переменные и их коммутационные соотношения для описания 

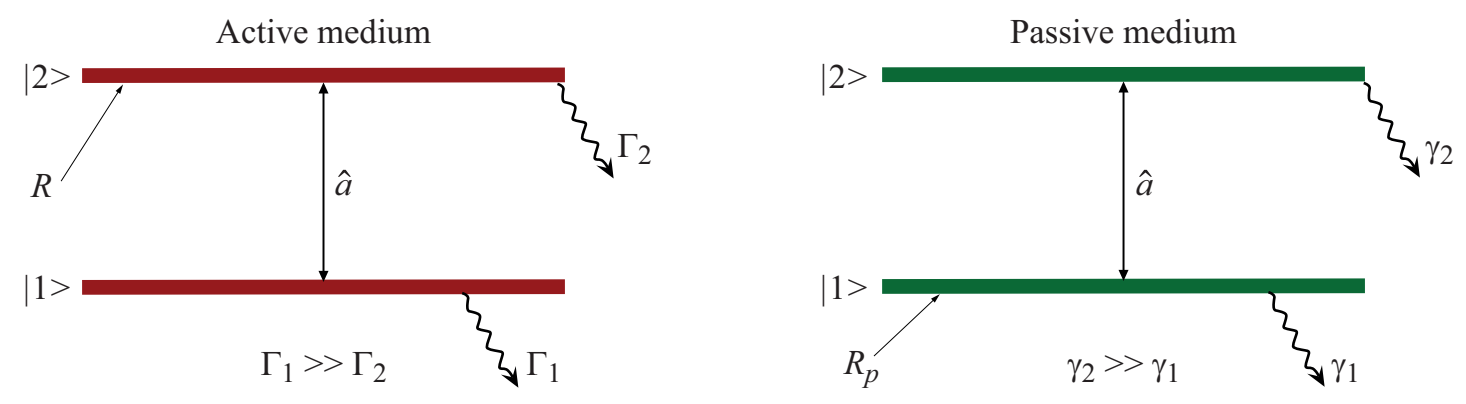

Рис. 1. Схема уровней активной и пасивной среды.

поля генерации и двухуровневой среды, содержащей активную и пассивную компоненты. В разд. 4 записываются уравнения Гейзенберга-Ланжевена для канонических переменных поля и вещества и произведен переход этих переменных в $c$-числовое представление. В разд. 5 получено замкнутое уравнение для полевой переменной в адиабатическом приближении. В разд. 6 обсуждено полуклассическое приближение для лазерного солитона. В разд. 7 приведены результаты моделирования синхронизации солитона с поддерживающим излучением. В Приложении А рассмотрена лазерная задача в приближении плоской волны. В Приложении В приведены ненулевые корреляционные функции в операторном и в $c$-числовом представлениях для поля и вещества.

\section{2. Физическая модель лазерного солитона}

Предполагается что в оптическом резонаторе бегущей волны с объёмом $V=S L(S-$ площадь поперечного сечения резонатора, $L$ - его длина) располагаются две разные среды (активная и пассивная), состоящие из двухуровневых атомов. Активная среда обеспечивает лазерную генерацию, амплитуда поля которой в дальнейшем описывается гейзенберговым оператором $\hat{a}$. Пассивная осуществляет насыщенное поглощение поля генерации. На рис. 1 показана энергетическая структура для атомов. Предполагается, что атомы обеих сред двухуровневые. Будем полагать, что верхний уровень активной среды возбуждается за счет некогерентной накачки со скоростью $R$. Одновременно пассивная среда возбуждается на нижний уровень со скоростью $R_{p}$.

Что касается оптического резонатора, то это резонатор бегущих плоских волн. Одна из волн, бегущих в положительном направлении оси $z$, является лазерной. Предполагается, что поперечный размер резонатора $\sqrt{S}$ много больше его длины $L$ так, что поперечные моды образуют сплошной спектр.

Изначально (до возникновения генерации) обе среды внутри резонатора не вносят каких-либо пространственных неоднородностей. Тем самым предполагается, что флуктуациями числа атомов мы пренебрегаем. В то же время в процессе лазерной генерации при рождении солитона возникает поперечная неоднородность, связанная с одновременным действием нелинейной фокусировки и дифракционным расплыванием лазерного луча.

\section{3. Канонические переменные для описания поля и двухуровневой среды в режиме лазерной генерации}

\section{1. Нормированная амплитуда лазерного солитона}

При классическом описании электромагнитного поля основными характеристиками считаются напряженности полей или их векторный и скалярный потенциалы. В рамках квантовой электродинамики предпочтительнее использовать канонические переменные. Это, с одной стороны, позволяет достаточно полно описать свойства поля, а с другой стороны, оказывается более наглядным и заметно облегчает математическую сторону вопроса.

Наше рассмотрение начнем с представления напряженности поля в виде разложения по полному набору собственных функций оптического резонатора. Пусть это будет набор бегущих волн. Таким образом, положительно-частотный оператор напряженности электрического поля внутри резонатора может быть записан в хорошо известном виде $[14,15]$

$$
\hat{E}(\mathbf{r}, t)=i\left(\frac{\hbar}{2 \varepsilon_{0} V}\right)^{1 / 2} \sum_{\mathbf{k}} \sqrt{\omega_{\mathbf{k}}} \hat{a}_{\mathbf{k}} e^{i \mathbf{k r}-i \omega_{\mathbf{k}} t} .
$$

Здесь $\hbar-$ приведенная постоянная Планка, $\varepsilon_{0}-$ линейная составляющая фоновой диэлектрической проницаемости, $\mathbf{k}$ - волновой вектор парциальных плоских волн и $\omega_{\mathbf{k}}$ - отвечающая ему частота. Эта запись может быть применена, разумеется, в классической и в квантовой электродинамике. Но в квантовой теории нормированные амплитуды $\hat{a}_{\mathbf{k}}$ подчиняются коммутационному соотношению $\left[\hat{a}_{\mathbf{k}}, \hat{a}_{\mathbf{k}^{\prime}}^{\dagger}\right]=\delta_{\mathbf{k}^{\prime}}$. Волновой вектор - индекс суммирования $\mathbf{k}$ - включает совокупность индексов 


$$
\begin{gathered}
k_{x}, k_{y}, k_{z}, \text { где } \\
k_{x}=\frac{2 \pi}{\sqrt{S}} n_{x}, \quad k_{y}=\frac{2 \pi}{\sqrt{S}} n_{y}, \quad k_{z}=\frac{2 \pi}{L} n_{z}, \\
n_{x}, n_{y}, n_{z}=0, \pm 1, \pm 2, \cdots .
\end{gathered}
$$

Величина $k_{z}$ нумерует плоские волны, бегущие в положительном и отрицательном направлениях оси $z$ (продольные волны). Поперечные волны будем описывать вектором $\mathbf{q}=\left(k_{x}, k_{y}\right)$ таким образом, что $\mathbf{k}=\mathbf{k}_{z}+\mathbf{q}$.

Формула (1) пригодна для описания различных ситуаций. Ограничимся случаем, когда поле внутри резонатора $\hat{E}(\mathbf{r}, t)$ может быть представлено в виде плоской монохроматической волны, бегущей в положительном направлении оси $z$ и слабо промодулированной в пространстве и времени. В этом случае напряженность поля внутри резонатора может быть записана в виде

$$
\begin{aligned}
\hat{E}(z, \boldsymbol{\rho}, t) & =i\left(\frac{\hbar \omega_{0}}{2 \varepsilon_{0} L}\right)^{1 / 2} e^{i k_{0} z-i \omega_{0} t} \hat{a}(z, \boldsymbol{\rho}, t), \\
\mathbf{r} & =(z, \boldsymbol{\rho}), \quad \boldsymbol{\rho}=(x, y) .
\end{aligned}
$$

В нашем рассмотрении величина $\hat{a}(z, \rho, t)$ является искомой канонической амплитудой поля, которая описывает медленное развитие поля в пространстве и времени. Нетрудно увидеть, что размерность амплитуды есть $\mathrm{cm}^{-1}$ и что физический смысл величины $\left\langle\hat{a}^{\dagger}(z, \boldsymbol{\rho}, t) \hat{a}(z, \boldsymbol{\rho}, t)\right\rangle$ - это среднее число фотонов, приходящихся на единичное поперечное сечение луча. Далее обсудим актуальные коммутационные соотношения для этой амплитуды и построим для нее управляющее уравнение.

Сравнивая формулы (1) и (3), сможем записать амплитуду $\hat{a}(z, \boldsymbol{\rho}, t)$ в виде суммы:

$$
\hat{a}(z, \boldsymbol{\rho}, t)=\frac{1}{\sqrt{S}} \sum_{\mathbf{k}} \sqrt{\omega_{\mathbf{k}} / \omega_{0}} \hat{a}_{\mathbf{k}} e^{i\left(k_{z}-k_{0}\right) z+i \mathbf{q} \boldsymbol{\rho}} e^{-i\left(\omega_{\mathbf{k}}-\omega_{0}\right) t}
$$

Полагаем, что из всех продольных мод за счет накачки лазерной среды возбуждается только одна мода с волновым числом $k_{z}=k_{0}$. Это означает, что в выражении (4) суммирование по полному набору $\mathbf{k}$ может быть заменено на суммирование только по поперечным модам в виде:

$$
\hat{a}(\boldsymbol{\rho}, t)=\frac{1}{\sqrt{S}} \sum_{\mathbf{q}} \sqrt{\omega_{\mathbf{q}} / \omega_{0}} \hat{a}_{\mathbf{q}} e^{i \mathbf{q} \boldsymbol{\rho}-i\left(\omega_{\mathbf{q}}-\omega_{0}\right) t} .
$$

Как видим, в одномодовом приближении амплитуда $\hat{a}$ не зависит от продольной координаты $z$. Это было бы не так, если бы мы имели дело с резонатором стоячих волн.

В дальнейшем будет важным знать коммутационное соотношение $\left[\hat{a}(\boldsymbol{\rho}, t), \hat{a}^{\dagger}\left(\boldsymbol{\rho}^{\prime}, t\right)\right]$, которое запишем на основе разложения (5) в виде

$$
\left[\hat{a}(\boldsymbol{\rho}, t), \hat{a}^{\dagger}\left(\boldsymbol{\rho}^{\prime}, t\right)\right]=\frac{1}{S} \sum_{\mathbf{q}} \omega_{\mathbf{q}} / \omega_{0} e^{i \mathbf{q}\left(\boldsymbol{\rho}-\boldsymbol{\rho}^{\prime}\right)}
$$

Пусть поперечный размер резонатора настолько большой, что поперечные моды образуют непрерывный спектр. Это обязывает нас заменить суммирование по поперечным модам на интегрирование согласно правилу

$$
\sum_{\mathbf{q}} \cdots \rightarrow \frac{S}{(2 \pi)^{2}} \int d^{2} \mathbf{q} \cdots
$$

Поскольку мы можем записать произвольный волновой вектор в виде суммы волновых векторов продольной и поперечной волн $\mathbf{k}=\mathbf{k}_{0}+\mathbf{q}$, то возведя это равенство в квадрат, получаем $k^{2}=k_{0}^{2}+q^{2}$. Тогда в параксиальном приближении $k_{0} \gg q$ можно записать

$$
\frac{\omega_{\mathbf{q}}}{\omega_{0}} \approx 1+\frac{q^{2}}{2 k_{0}^{2}}
$$

Заменяя в формуле (6) суммирование на интегрирование согласно правилу (7), получаем возможность выполнить это интегрирование и вычислить следующее коммутационное соотношение для канонической амплитуды поля:

$$
\left[\hat{a}(\boldsymbol{\rho}, t), \hat{a}^{\dagger}\left(\boldsymbol{\rho}^{\prime}, t\right)\right]=\tilde{\delta}\left(\boldsymbol{\rho}-\boldsymbol{\rho}^{\prime}\right) .
$$

Здесь обозначено

$$
\begin{gathered}
\tilde{\delta}\left(\boldsymbol{\rho}-\boldsymbol{\rho}^{\prime}\right)=\left(1-\frac{1}{2 k_{0}^{2}} \Delta_{\perp}\right) \delta^{2}\left(\boldsymbol{\rho}-\boldsymbol{\rho}^{\prime}\right), \\
\Delta_{\perp}=\frac{\partial^{2}}{\partial x^{2}}+\frac{\partial^{2}}{\partial y^{2}} .
\end{gathered}
$$

Покажем, что гамильтониан поля может быть выражен через амплитуду $\hat{a}$. Будем исходить из хорошо известной формулы для энергии поля, выраженной через электрическую $\hat{E}(\mathbf{r}, t)$ и магнитную $\hat{H}(\mathbf{r}, t)$ напряженности:

$$
\begin{aligned}
& \hat{H}_{\text {field }}=\frac{1}{2} \int_{V} d^{3} r\left[\varepsilon_{0}(\hat{E}(\mathbf{r}, t)+H . c .)^{2}\right. \\
& \left.\quad+\mu_{0}(\hat{H}(\mathbf{r}, t)+H . c .)^{2}\right]=\int_{V} d^{3} r \varepsilon_{0}(\hat{E}(\mathbf{r}, t)+H . c .)^{2} \\
& \quad \approx \int_{V} d^{3} r \varepsilon_{0}\left[\hat{E}^{\dagger}(\mathbf{r}, t) \hat{E}(\mathbf{r}, t)+\hat{E}(\mathbf{r}, t) \hat{E}^{\dagger}(\mathbf{r}, t)\right] .
\end{aligned}
$$

Здесь $\mu_{0}$ - магнитная проницаемость среды и Н.с. означает эрмитово сопряжение. Мы учли, во-первых, что для свободного поля вклад от электрического поля совпадает с вкладом от магнитного поля. Во-вторых, приняли приближение вращающейся волны, согласно которому под знаком интегрирования пренебрегается вкладом от быстрых членов по сравнению с медленными. При учете (3), (4) последнее равенство переписывается через гейзенбергову амплитуду поля $\hat{a}$ в виде

$$
\hat{H}_{\text {field }}=\frac{\hbar \omega_{0}}{2} \int_{S} d^{2} \rho\left[\hat{a}^{\dagger}(\boldsymbol{\rho}, t) \hat{a}(\boldsymbol{\rho}, t)+\hat{a}(\boldsymbol{\rho}, t) \hat{a}^{\dagger}(\boldsymbol{\rho}, t)\right] .
$$


Теперь, когда ввели в рассмотрение каноническую амплитуду поля (вместе с коммутационным соотношением) и выразили через эту амплитуду гамильтониан поля, мы можем, применяя обычные правила квантовой механики, записать уравнение Гейзенберга для амплитуды поля $\hat{a}(\boldsymbol{\rho}, t)$ в виде

$$
\frac{\partial}{\partial t} \hat{a}(\boldsymbol{\rho}, t)=i \omega_{0} \hat{a}(\boldsymbol{\rho}, t)+\frac{i}{\hbar}\left[\hat{H}_{\text {field }}, \hat{a}(\boldsymbol{\rho}, t)\right] .
$$

Здесь первое слагаемое справа возникло из-за того, что амплитуда $\hat{a}(\boldsymbol{\rho}, t)$ развивается во времени медленно, поскольку при ее определении был выделен множитель, колеблющийся на оптической частоте $\omega_{0}$.

Принимая во внимание коммутационное соотношение (9), получим уравнение для канонической амплитуды поля в пустом резонаторе:

$$
\frac{\partial \hat{a}(\boldsymbol{\rho}, t)}{\partial t}-\frac{i c}{2 k_{0}} \Delta_{\perp} \hat{a}(\boldsymbol{\rho}, t)=0 .
$$

Далее увидим, что это уравнение допускает решение для поля не только в виде плоской волны, как это могло ожидаться в случае одномодового приближения, но и в виде некоторой поперечно локализованной структуры, которую принято называть солитоном.

\section{2. Операторы коллективных заселенностей для двухуровневой многоатомной среды}

Гамильтониан изолированной двухуровневой среды может быть записан в виде суммы гамильтонианов для одиночных атомов в следующим виде

$$
\begin{aligned}
\hat{H}_{\text {matter }}= & W_{2} \sum_{j} \hat{\sigma}_{2}^{j}\left(\mathbf{r}_{j}, t\right) \Theta\left(t-t_{j}\right) \\
& +W_{1} \sum_{j} \hat{\sigma}_{1}^{j}\left(\mathbf{r}_{j}, t\right) \Theta\left(t-t_{j}\right) .
\end{aligned}
$$

Здесь суммирование по $j$ означает суммирование по индивидуальным атомам, а $t_{j}$ - это момент появления $j$-го атома на одном из уровней с энергиями $W_{1}$ или $W_{2}$ $\left(W_{2}-W_{1}=\hbar \omega_{a}\right)$. Это обеспечивается некоторой схемой некогерентного возбуждения среды, которая задается набором функций $\Theta\left(t-t_{j}\right)$, которые равны нулю при $t<t_{j}$ и единице при $t>t_{j}$. Положения атомов внутри резонатора определяются вектором $\mathbf{r}_{j}$, атомы неподвижны и не взаимодействуют друг с другом. Проекционные операторы для $j$-го атома задаются в виде $\hat{\sigma}_{2}^{j}\left(\mathbf{r}_{j}, t\right)=|2\rangle_{j j}\langle 2|$ для верхнего уровня и в виде $\hat{\sigma}_{1}^{j}\left(\mathbf{r}_{j}, t\right)=|1\rangle_{j j}\langle 1|$ для нижнего уровня.

Формула (15) может быть переписана в виде

$$
\begin{aligned}
\hat{H}_{\text {matter }}= & \int_{V} d^{3} r W_{2} \sum_{j} \hat{\sigma}_{2}^{j}(\mathbf{r}, t) \Theta\left(t-t_{j}\right) \delta^{3}\left(\mathbf{r}-\mathbf{r}_{j}\right) \\
& +\int_{V} d^{3} r W_{1} \sum_{j} \hat{\sigma}_{1}^{j}(\mathbf{r}, t) \Theta\left(t-t_{j}\right) \delta^{3}\left(\mathbf{r}-\mathbf{r}_{j}\right) .
\end{aligned}
$$

Будем полагать, что среда вдоль оси $z$ распределена однородно в любой момент времени. Напоминаем, что до начала процесса генерации среда внутри резонатора предполагалась однородной по всем трем направлениям. Однако в силу специфических условий лазерной генерации при учете дифракционного расплывания лазерного луча в поперечном направлении возникает неоднородность поля. Как следствие, возникает соответствующая неоднородность среды, т.е. зависимость от переменной $\boldsymbol{\rho}$. Однородность среды вдоль оси $z$ сохраняется в силу одномодового приближения. Это означает,что мы можем пренебречь зависимостью атомных переменных от продольной координаты $z$ :

$$
\begin{gathered}
\hat{\sigma}_{2}^{j}(\mathbf{r}, t)=\hat{\sigma}_{2}^{j}(z, \boldsymbol{\rho}, t) \rightarrow \hat{\sigma}_{2}^{j}(\boldsymbol{\rho}, t), \\
\hat{\sigma}_{1}^{j}(\mathbf{r}, t)=\hat{\sigma}_{1}^{j}(z, \boldsymbol{\rho}, t) \rightarrow \hat{\sigma}_{1}^{j}(\boldsymbol{\rho}, t) .
\end{gathered}
$$

В выражении (16) возьмем интеграл по переменной $z$, но сохраняем интегралы по поперечным координатам. В результате получим следующее выражение

$$
\begin{aligned}
\hat{H}_{\text {matter }}= & W_{2} \int_{S} d^{2} \rho \sum_{j} \hat{\sigma}_{2}^{j}(\boldsymbol{\rho}, t) \Theta\left(t-t_{j}\right) \delta^{2}\left(\boldsymbol{\rho}-\boldsymbol{\rho}_{j}\right) \\
& +W_{1} \int_{S} d^{2} \rho \sum_{j} \hat{\sigma}_{1}^{j}(\boldsymbol{\rho}, t) \Theta\left(t-t_{j}\right) \delta^{2}\left(\boldsymbol{\rho}-\boldsymbol{\rho}_{j}\right) .
\end{aligned}
$$

Введем в рассмотрение операторы коллективных плотностей заселенностей:

$$
\begin{gathered}
\hat{\sigma}_{1}(\boldsymbol{\rho}, t)=\sum_{j} \hat{\sigma}_{1}^{j}(\boldsymbol{\rho}, t) \Theta\left(t-t_{j}\right) \delta^{2}\left(\boldsymbol{\rho}-\boldsymbol{\rho}_{j}\right), \\
\hat{\sigma}_{1}^{j}(\boldsymbol{\rho}, t)=|1\rangle_{j j}\langle 1|, \\
\hat{\sigma}_{2}(\boldsymbol{\rho}, t)=\sum_{j} \hat{\sigma}_{2}^{j}(\boldsymbol{\rho}, t) \Theta\left(t-t_{j}\right) \delta^{2}\left(\boldsymbol{\rho}-\boldsymbol{\rho}_{j}\right), \\
\hat{\sigma}_{2}^{j}(\boldsymbol{\rho}, t)=|2\rangle_{j j}\langle 2| .
\end{gathered}
$$

Размерность этих величин $\mathrm{cm}^{-2}$, они имеют физический смысл числа атомов на единичном поперечном сечении. Перепишем через них гамильтониан:

$$
\hat{H}_{\text {matter }}^{(a)}=\int_{S} d^{2} \rho\left[W_{2} \hat{\sigma}_{2}(\boldsymbol{\rho}, t)+W_{1} \hat{\sigma}_{1}(\boldsymbol{\rho}, t)\right] .
$$

Здесь мы добавили у гамильтониана индекс $(a)$, поясняющий, что формула записана для активной среды. Точно также можно записать гамильтониан для свободной пассивной среды:

$$
\hat{H}_{\text {matter }}^{(p)}=\int_{S} d^{2} \rho\left[W_{2} \hat{\pi}_{2}(\boldsymbol{\rho}, t)+W_{1} \hat{\pi}_{1}(\boldsymbol{\rho}, t)\right] .
$$

Здесь коллективные переменные вводятся точно так же, как и для активной среды, и имеют вид

$$
\hat{\pi}_{1}(\boldsymbol{\rho}, t)=\sum_{j} \hat{\pi}_{1}^{j}(\boldsymbol{\rho}, t) \Theta\left(t-t_{j}\right) \delta^{2}\left(\boldsymbol{\rho}-\boldsymbol{\rho}_{j}\right),
$$




$$
\begin{gathered}
\hat{\pi}_{1}^{j}(\boldsymbol{\rho}, t)=|1\rangle_{j j}\langle 1|, \\
\hat{\pi}_{2}(\boldsymbol{\rho}, t)=\sum_{j} \hat{\pi}_{2}^{j}(\boldsymbol{\rho}, t) \Theta\left(t-t_{j}\right) \delta^{2}\left(\boldsymbol{\rho}-\boldsymbol{\rho}_{j}\right), \\
\hat{\pi}_{2}^{j}(\boldsymbol{\rho}, t)=|2\rangle_{j j}\langle 2| .
\end{gathered}
$$

\section{3. Дипольное взаимодействие поля со средой внутри резонатора: операторы коллективной когерентности}

Физическая система, которую мы хотим рассмотреть, представляет собой оптический высокодобротный резонатор, который равномерно заполнен двумя атомными двухуровневыми средами разного предназначения. Активная среда обеспечивает продольно одномодовую лазерную генерацию, а пассивная осуществляет насыщенное поглощение этой генерации.

Ограничиваясь дипольным приближением во взаимодействии поля с каждым из двухуровневых атомов, мы сможем записать гамильтониан взаимодействия поля и вещества в виде суммы двух гамильтонианов $\hat{V}=\hat{V}^{(a)}+\hat{V}^{(p)}$ соответственно для активной и пассивной сред. Сначала обсудим в деталях взаимодействие с активной средой:

$$
\begin{aligned}
\hat{V}^{(a)}= & -\sum_{j}\left(\hat{d}^{j}\left(\mathbf{r}_{j}, t\right)+\hat{d}^{j \dagger}\left(\mathbf{r}_{j}, t\right)\right) \\
& \times\left(\hat{E}\left(\mathbf{r}_{j}, t\right)+\hat{E}^{\dagger}\left(\mathbf{r}_{j}, t\right)\right) \Theta\left(t-t_{j}\right) .
\end{aligned}
$$

Для величины $\hat{E}$ мы должны взять выражение (3), полагая там, что каждый атом „видит“поле в точке расположения атома, и принимая во внимание, что задача однородна вдоль оси $z$.

Оператор

$$
\hat{d}^{j}=\mu \hat{\sigma}^{j} e^{-i \omega_{a} t}, \quad \hat{\sigma}^{j}=|1\rangle_{j j}\langle 2|
$$

имеет смысл оператора дипольного момента $j$-го атома, $\mu-$ матричный элемент дипольного момента лазерного перехода, $\omega_{a}-$ частота лазерного перехода в активной среде.

В приближении вращающихся волн выражение (25) переписывается как

$$
\begin{aligned}
\hat{V}^{(a)} \approx & -\sum_{j}\left(\hat{d}^{j}\left(\mathbf{r}_{j}, t\right) \hat{E}^{\dagger}\left(\mathbf{r}_{j}, t\right)\right. \\
& \left.+\hat{E}\left(\mathbf{r}_{j}, t\right) \hat{d}^{j \dagger}\left(\mathbf{r}_{j}, t\right)\right) \Theta\left(t-t_{j}\right) .
\end{aligned}
$$

Подставляя сюда формулы (4) и (25), получим

$$
\begin{aligned}
\hat{V}^{(a)}= & -i \hbar g \sum_{j} \hat{\sigma}^{j}\left(\boldsymbol{\rho}_{j}, t\right) \hat{a}^{\dagger}\left(\boldsymbol{\rho}_{j}, t\right) \Theta\left(t-t_{j}\right) \\
& \times e^{-i k_{0} z_{j}+i \delta_{a} t}+H . c ., \quad g=\mu_{a}\left(\frac{\omega_{0}}{2 \varepsilon_{0} \hbar L}\right)^{1 / 2} .
\end{aligned}
$$

Здесь $g-$ константа взаимодействия поля и активного вещества в дипольном приближении, $\delta_{a}=\omega_{0}-\omega_{a}-$ частотная отстройка активной среды от продольной моды резонатора. Это теперь нетрудно переписать через коллективную когерентность, которую определим в виде

$$
\begin{aligned}
\hat{\sigma}(\boldsymbol{\rho}, t)= & -i \sum_{j} \hat{\sigma}^{j}(\boldsymbol{\rho}, t) \Theta\left(t-t_{j}\right) \delta^{2}\left(\boldsymbol{\rho}-\boldsymbol{\rho}_{j}\right) \\
& \times e^{-i k_{0} z_{j}+i \delta_{a} t}, \quad \hat{\sigma}^{j}\left(\boldsymbol{\rho}_{j}, t\right)=|1\rangle_{j j}\langle 2| .
\end{aligned}
$$

Гамильтониан взаимодействия при этом имеет вид

$$
\hat{V}^{(a)}=\hbar g \int_{S} d^{2} \rho\left[\hat{\sigma}(\boldsymbol{\rho}, t) \hat{a}^{\dagger}(\boldsymbol{\rho}, t)+\hat{\sigma}^{\dagger}(\boldsymbol{\rho}, t) \hat{a}(\boldsymbol{\rho}, t)\right] .
$$

Рассуждая подобным образом, можем ввести в рассмотрение коллективную когерентность пассивной среды $\hat{\pi}$ и переписать через нее гамильтониан взаимодействия лазерного поля с пассивной средой:

$$
\begin{gathered}
\hat{V}^{(p)}=\hbar g_{p} \int_{S} d^{2} \rho\left[\hat{\pi}(\boldsymbol{\rho}, t) \hat{a}^{\dagger}(\boldsymbol{\rho}, t)+\hat{\pi}^{\dagger}(\boldsymbol{\rho}, t) \hat{a}(\boldsymbol{\rho}, t)\right], \\
g_{p}=\mu_{p}\left(\frac{\omega_{0}}{2 \varepsilon_{0} \hbar L}\right)^{1 / 2} .
\end{gathered}
$$

Здесь оператор коллективной когерентности $\hat{\pi}$ определен в виде

$$
\begin{gathered}
\hat{\pi}(\boldsymbol{\rho}, t)=-i \sum_{j} \hat{\pi}^{j}(\boldsymbol{\rho}, t) \Theta\left(t-t_{j}\right) \delta^{2}\left(\boldsymbol{\rho}-\boldsymbol{\rho}_{j}\right) e^{-i k_{0} z_{j}+i \delta_{p} t}, \\
\hat{\pi}^{j}\left(\boldsymbol{\rho}_{j}, t\right)=|1\rangle_{j j}\langle 2| .
\end{gathered}
$$

Это определение совпадает с определением оператора $\hat{\sigma}$. Частотный сдвиг поля от атомного перехода пассивной среды определяется в виде $\delta_{p}=\omega_{0}-\omega_{p}$. Мы снова относим соответствующий множитель в определение коллективной когерентности.

\section{4. Коммутационные соотношения для коллективных атомных операторов}

Согласно предыдущему изложению, наша система в рамках квантовых представлений определяется следующим набором канонических амплитуд. Во-первых, это амплитуда лазерной генерации $\hat{a}(\rho, t)$. Во-вторых, это набор коллективных атомных операторов для двух резонансных сред: коллективная когерентность активной среды $\hat{\sigma}(\boldsymbol{\rho}, t)$, коллективные заселенности активной среды нижнего $\hat{\sigma}_{1}(\boldsymbol{\rho}, t)$ и верхнего атомных уровней $\hat{\sigma}_{2}(\boldsymbol{\rho}, t)$. Аналогично, для пассивной среды имеется набор переменных $\hat{\pi}(\boldsymbol{\rho}, t), \hat{\pi}_{1}(\boldsymbol{\rho}, t), \hat{\pi}_{2}(\boldsymbol{\rho}, t)$. Для этих семи амплитуд мы должны написать семь гейзенберговых уравнений.

Для того чтобы иметь возможность проводить вычисления с этими операторами, следует указать для них актуальные коммутационные соотношения. Для амплитуды поля мы записали это соотношение в виде (9)-(10). 
Выше в выражениях (19), (20), (23), (24) и (29)-(32) мы сформировали коллективные переменные как линейную комбинацию индивидуальных переменных. Это дает возможность выразить коммутационные соотношения для коллективных переменных через коммутационные соотношения для индивидуальных переменных:

$$
\begin{gathered}
{\left[\hat{\sigma}^{j}\left(\boldsymbol{\rho}_{j}, t\right), \hat{\sigma}^{j \dagger}\left(\boldsymbol{\rho}_{j}, t\right)\right]=\hat{\sigma}_{1}^{j}\left(\boldsymbol{\rho}_{j}, t\right)-\hat{\sigma}_{2}^{j}\left(\boldsymbol{\rho}_{j}, t\right),} \\
{\left[\hat{\sigma}_{1}^{j}\left(\boldsymbol{\rho}_{j}, t\right), \hat{\sigma}^{j}\left(\boldsymbol{\rho}_{j}, t\right)\right]=\hat{\sigma}^{j}\left(\boldsymbol{\rho}_{j}, t\right),} \\
{\left[\hat{\sigma}_{2}^{j}\left(\boldsymbol{\rho}_{j}, t\right), \hat{\sigma}^{j}\left(\boldsymbol{\rho}_{j}, t\right)\right]=-\hat{\sigma}^{j}\left(\boldsymbol{\rho}_{j}, t\right) .}
\end{gathered}
$$

Тогда для коллективных переменных нетрудно получить ненулевые коммутационные соотношения:

$$
\begin{gathered}
{\left[\hat{\sigma}(\boldsymbol{\rho}, t), \hat{\sigma}^{\dagger}\left(\boldsymbol{\rho}^{\prime}, t\right)\right]=\left[\hat{\sigma}_{1}(\boldsymbol{\rho}, t)-\hat{\sigma}_{2}(\boldsymbol{\rho}, t)\right] \delta^{2}\left(\boldsymbol{\rho}-\boldsymbol{\rho}^{\prime}\right),} \\
{\left[\hat{\sigma}_{1}(\boldsymbol{\rho}, t), \hat{\sigma}\left(\boldsymbol{\rho}^{\prime}, t\right)\right]=\hat{\sigma}(\boldsymbol{\rho}, t) \delta^{2}\left(\boldsymbol{\rho}-\boldsymbol{\rho}^{\prime}\right),} \\
{\left[\hat{\sigma}_{2}(\boldsymbol{\rho}, t), \hat{\sigma}\left(\boldsymbol{\rho}^{\prime}, t\right)\right]=-\hat{\sigma}(\boldsymbol{\rho}, t) \delta^{2}\left(\boldsymbol{\rho}-\boldsymbol{\rho}^{\prime}\right) .}
\end{gathered}
$$

Точно также можем записать аналогичные выражения для пассивной среды. В этом случае коллективные переменные оказываются определенными следующим образом:

$$
\begin{gathered}
{\left[\hat{\pi}(\boldsymbol{\rho}, t), \hat{\pi}^{\dagger}\left(\boldsymbol{\rho}^{\prime}, t\right)\right]=\left[\hat{\pi}_{1}(\boldsymbol{\rho}, t)-\hat{\pi}_{2}(\boldsymbol{\rho}, t)\right] \delta^{2}\left(\boldsymbol{\rho}-\boldsymbol{\rho}^{\prime}\right),} \\
{\left[\hat{\pi}_{1}(\boldsymbol{\rho}, t), \hat{\pi}\left(\boldsymbol{\rho}^{\prime}, t\right)\right]=\hat{\pi}(\boldsymbol{\rho}, t) \delta^{2}\left(\boldsymbol{\rho}-\boldsymbol{\rho}^{\prime}\right),} \\
{\left[\hat{\pi}_{2}(\boldsymbol{\rho}, t), \hat{\pi}\left(\boldsymbol{\rho}^{\prime}, t\right)\right]=-\hat{\pi}(\boldsymbol{\rho}, t) \delta^{2}\left(\boldsymbol{\rho}-\boldsymbol{\rho}^{\prime}\right) .}
\end{gathered}
$$

\section{4. Система уравнений Гейзенберга-Ланжевена}

\section{1. Уравнения Гейзенберга}

Лазерная система, которую мы здесь рассматриваем, является открытой и потому существенно зависящей от разного рода релаксационных процессов и процессов возбуждения. Тем не менее на первой стадии вывода уравнений мы игнорируем эти процессы. Понятно, что полученные таким образом уравнения не могут быть полноценными, однако, хорошо известно, каким образом возможно исправить ситуацию. Мы можем феноменологически ввести в уравнения нужные члены. Этот подход может быть относительно легко оправдан аналитически [16].

Запишем сначала уравнения Гейзенберга для амплитуд поля и вещества, имея в виду хорошо известные равенства

$$
\begin{gathered}
\frac{\partial}{\partial t} \hat{a}=i \omega_{0} \hat{a}(\boldsymbol{\rho}, t)+\frac{i}{\hbar}\left[\hat{H}_{\text {field }}+\hat{V}^{(a)}+\hat{V}^{(p)}, \hat{a}\right], \\
\frac{\partial}{\partial t} \hat{\sigma}=i \omega_{a} \hat{\sigma}+\frac{i}{\hbar}\left[\hat{H}_{\text {matter }}^{(a)}+\hat{V}^{(a)}, \hat{\sigma}\right], \\
\frac{\partial}{\partial t} \hat{\pi}=i \omega_{p} \hat{\pi}(\boldsymbol{\rho}, t)+\frac{i}{\hbar}\left[\hat{H}_{\text {matter }}^{(p)}+\hat{V}^{(p)}, \hat{\pi}\right],
\end{gathered}
$$

$$
\begin{gathered}
\frac{\partial}{\partial t} \hat{\sigma}_{1}=\frac{i}{\hbar}\left[\hat{H}_{\text {matter }}^{(a)}+\hat{V}^{(a)}, \hat{\sigma}_{1}\right], \\
\frac{\partial}{\partial t} \hat{\sigma}_{2}=\frac{i}{\hbar}\left[\hat{H}_{\text {matter }}^{(a)}+\hat{V}^{(a)}, \hat{\sigma}_{2}\right], \\
\frac{\partial}{\partial t} \hat{\pi}_{1}=\frac{i}{\hbar}\left[\hat{H}_{\text {matter }}^{(p)}+\hat{V}^{(p)}, \hat{\pi}_{1}\right] . \\
\frac{\partial}{\partial t} \hat{\pi}_{2}=\frac{i}{\hbar}\left[\hat{H}_{\text {matter }}^{(p)}+\hat{V}^{(p)}, \hat{\pi}_{2}\right] .
\end{gathered}
$$

Теперь имеем возможность, согласно стандартным правилам квантовой механики, записать уравнения Гейзенберга для всех канонических переменных, используя полученные выше коммутационные соотношения и гамильтониан. После вычисления актуальных коммутаторов уравнения приобретают вид

$$
\begin{gathered}
\frac{\partial \hat{a}}{\partial t}-\frac{i c}{2 k_{0}} \Delta_{\perp} \hat{a}=g \hat{\sigma}+g_{p} \hat{\pi}, \\
\frac{\partial \hat{\sigma}}{\partial t}=i \delta_{a} \hat{\sigma}+g\left(\hat{\sigma}_{2}-\hat{\sigma}_{1}\right) \hat{a}, \\
\frac{\partial \hat{\sigma}_{1}}{\partial t}=g\left(\hat{\sigma}^{\dagger} \hat{a}+\hat{\sigma} \hat{a}^{\dagger}\right), \\
\frac{\partial \hat{\sigma}_{2}}{\partial t}=-g\left(\hat{\sigma}^{\dagger} \hat{a}+\hat{a}^{\dagger} \hat{\sigma}\right), \\
\frac{\partial \hat{\pi}}{\partial t}=i \delta_{p} \hat{\pi}+g_{p}\left(\hat{\pi}_{2}-\hat{\pi}_{1}\right) \hat{a}, \\
\frac{\partial \hat{\pi}_{1}}{\partial t}=g_{p}\left(\hat{\pi}^{\dagger} \hat{a}+\hat{a}^{\dagger} \hat{\pi}\right), \\
\frac{\partial \hat{\pi}_{2}}{\partial t}=-g_{p}\left(\hat{\pi}^{\dagger} \hat{a}+\hat{\pi} \hat{a}^{\dagger}\right) .
\end{gathered}
$$

\section{2. Включение в схему релаксационных процессов: операторное представление}

Уравнения (49)-(55) записаны в пренебрежении какими-либо релаксационными процессами и в пренебрежении возбуждениями активной и пассивной сред извне. Между тем любая лазерная система существенно зависит от этих процессов независимо от того, имеем ли мы дело с классическим или квантовым описанием.

Хорошо известно, каким образом нужно действовать, чтобы учесть эти важные аспекты на уровне дифференциальных уравнений. Например, чтобы учесть затухание поля из резонатора со скоростью $\kappa$, в правой части уравнения (49) вводится слагаемое, ответственное за это затухание и имеющее вид $-1 / 2 \kappa \hat{a}$. Как известно, в квантовой теории мы должны при этом ввести в уравнение ланжевеновский источник. Точно также следует поступить со всеми другими уравнениями (50)-(55), принимая во внимание, что константы $\Gamma, \Gamma_{1}, \Gamma_{2}-$ это скорости затухания когерентности активной среды и заселенностей нижнего и верхнего уровней. Соответственно для пассивной среды имеет место набор констант затухания $\gamma, \gamma_{1}, \gamma_{2}$.

В нашей открытой системе следует также учесть некогерентное широкополосное возбуждение верхнего 
атомного уровня активной среды со скоростью $R_{a}$ и нижнего уровня пассивной среды со скоростью $R_{p}$. Соответствующие неоднородные члены вводятся в уравнения (52) и (54).

Проделав эти манипуляции, запишем уравнения в следующим виде. Для электромагнитного поля

$$
\frac{\partial \hat{a}}{\partial t}-\frac{i c}{2 k_{0}} \Delta_{\perp} \hat{a}=-\kappa / 2 \hat{a}+g \hat{\sigma}+g_{p} \hat{\pi}+\hat{F}_{a} .
$$

Соответствующие уравнения имеют место для активной среды:

$$
\begin{gathered}
\frac{\partial \hat{\sigma}}{\partial t}=-\left(\Gamma-i \delta_{a}\right) \hat{\sigma}+g\left(\hat{\sigma}_{2}-\hat{\sigma}_{1}\right) \hat{a}+\hat{F}, \\
\Gamma=1 / 2\left(\Gamma_{1}+\Gamma_{2}\right), \\
\frac{\partial \hat{\sigma}_{1}}{\partial t}=-\Gamma_{1} \hat{\sigma}_{1}+g\left(\hat{\sigma}^{\dagger} \hat{a}+\hat{\sigma} \hat{a}^{\dagger}\right)+\hat{F}_{1}, \\
\frac{\partial \hat{\sigma}_{2}}{\partial t}=R_{a}-\Gamma_{2} \hat{\sigma}_{2}-g\left(\hat{\sigma}^{\dagger} \hat{a}+\hat{a}^{\dagger} \hat{\sigma}\right)+\hat{F}_{2},
\end{gathered}
$$

и для пассивной среды:

$$
\begin{gathered}
\frac{\partial \hat{\pi}}{\partial t}=-\left(\gamma-i \delta_{p}\right) \hat{\pi}+g_{p}\left(\hat{\pi}_{2}-\hat{\pi}_{1}\right) \hat{a}+\hat{G}, \\
\gamma=1 / 2\left(\gamma_{1}+\gamma_{2}\right) \\
\frac{\partial \hat{\pi}_{1}}{\partial t}=R_{p}-\gamma_{1} \hat{\pi}_{1}+g_{p}\left(\hat{\pi}^{\dagger} \hat{a}+\hat{a}^{\dagger} \hat{\pi}\right)+\hat{G}_{1}, \\
\frac{\partial \hat{\pi}_{2}}{\partial t}=-\gamma_{2} \hat{\pi}_{2}-g_{p}\left(\hat{\pi}^{\dagger} \hat{a}+\hat{\pi} \hat{a}^{\dagger}\right)+\hat{G}_{2} .
\end{gathered}
$$

Таким образом мы превратили уравнения Гейзенберга в уравнения Гейзенберга-Ланжевена. При этом учли, что верхний уровень активной двухуровневой среды возбуждается некогерентно со средней скоростью $R_{a}$. В то же время учли и то, что оба лазерных уровня спонтанно распадаются на некоторые посторонние уровни. Верхний уровень распадается со скоростью $\Gamma_{2}$, а нижний со скоростью $\Gamma_{1}$. Что касается пассивной двухуровневой среды, то ее нижний уровень возбуждается извне со скоростью $R_{p}$, и оба уровня спонтанно распадаются на некоторые третьи уровни: верхний со скоростью $\gamma_{2}$ и нижний со скоростью $\gamma_{1}$.

Что касается ланжевеновских источников, в Приложении В представлены их ненулевые парные корреляции. Эти соотношения для источников активной среды $\hat{F}_{a}$ и $\hat{F}, \hat{F}_{1}, \hat{F}_{2}$ были получены в работе [17] на основе флуктуационно-диссипационной теоремы. Точно также мы провели вычисления корреляционных функций для пассивной среды, т.е. для ланжевеновских источников $\hat{G}, \hat{G}_{1}, \hat{G}_{2}$.

\section{3. $\quad$-числовое представление}

Полученные в предыдущем разделе операторные уравнения являются той основой, на которой может быть проведено дальнейшее развитие теории солитона. Однако, как известно, операторное представление существенно осложняет действия с ним. Поэтому, когда возможно, мы стремимся так или иначе уйти от операторного описания. В наших рассуждениях это возможно сделать, для чего имеется специально разработанная процедура, позволяющая перейти к так называемому $c$-числовому представлению.

Указанная процедура перехода к $c$-числовому представлению формулируется следующим образом. Вопервых, имея в виду актуальные коммутационные соотношения, мы должны привести к нормальному порядку все имеющие место в уравнениях (57)-(62) произведения операторов и затем превратить все операторы в $c$-числовые функции. Нормальное упорядочение операторов определено в виде $\hat{a}^{\dagger} \hat{\sigma}^{\dagger} \hat{\pi}^{\dagger} \hat{\sigma}_{2} \hat{\pi}_{2} \hat{\sigma}_{1} \hat{\pi}_{1} \hat{\pi} \hat{\sigma} \hat{a}$ [16]. Во-вторых, следует помнить, что новые $c$-числовые уравнения пригодны только для вычисления нормально упорядоченных средних величин. В-третьих, ланжевеновские источники должны быть переформулированы для нормально упорядоченных парных средних.

В нашем случае упорядочение произведений операторов справа в уравнениях $(57)-(62)$ достигаются простой их перестановкой. Например, в уравнении (57) справа выражение $\left(\hat{\sigma}_{2}-\hat{\sigma}_{1}\right) \hat{a}$ просто заменяется на выражение $\hat{a}\left(\hat{\sigma}_{2}-\hat{\sigma}_{1}\right)$, поскольку мы следим за операторами в один и тот же момент времени, и они при этом оказываются коммутирующими.

После подобных перестановок получаем $c$-числовые уравнения

$$
\begin{gathered}
\frac{\partial a}{\partial t}-\frac{i c}{2 k_{0}} \Delta_{\perp} a=-\kappa / 2 a+g \sigma+g_{p} \pi, \\
\frac{\partial \sigma}{\partial t}=-\left(\Gamma-i \delta_{a}\right) \sigma+g\left(\sigma_{2}-\sigma_{1}\right) a+F \\
\frac{\partial \sigma_{1}}{\partial t}=-\Gamma_{1} \sigma_{1}+g\left(\sigma^{*} \hat{a}+\sigma a^{*}\right)+F_{1}, \\
\frac{\partial \sigma_{2}}{\partial t}=R_{a}-\Gamma_{2} \sigma_{2}-g\left(\sigma^{*} a+a^{*} \sigma\right)+F_{2}, \\
\frac{\partial \pi}{\partial t}=-\left(\gamma-i \delta_{p}\right) \pi+g_{p}\left(\pi_{2}-\pi_{1}\right) a+G, \\
\frac{\partial \pi_{1}}{\partial t}=R_{p}-\gamma_{1} \pi_{1}+g_{p}\left(\pi^{*} a+a^{*} \pi\right)+G_{1}, \\
\frac{\partial \pi_{2}}{\partial t}=-\gamma_{2} \pi_{2}-g_{p}\left(\pi^{*} a+\pi a^{*}\right)+G_{2} .
\end{gathered}
$$

Как видим, внешний вид уравнений сохраняется, но разница возникает в свойствах ланжевеновских источников. Например, хорошо известно, что при переписывании уравнения (56) ланжевеновский источник $\hat{F}_{a}$ становится равным нулю. Нужно отметить, что это 
точный результат, и он не означает, что мы пренебрегли источником шума квантового поля. Все другие источники определены в Приложении В и по своим корреляционным свойствам совпадают с полученными в работе [17].

\section{5. Основное уравнение в адиабатическом приближении}

Вообще говоря, уравнения (63)-(69) полностью описывают лазерную систему, включая вариант с солитоном. Далее будем полагать, что полевая переменная развивается значительно медленнее, чем атомные. Это может быть обеспечено требованиями

$$
\kappa \ll \Gamma, \Gamma_{1}, \Gamma_{2}, \quad \kappa \ll \gamma, \gamma_{1}, \gamma_{2} .
$$

Это предположение позволит нам адиабатически исключить из рассмотрения атомные переменные и сконструировать замкнутое уравнение для полевой переменной. Кроме того, положим, что

$$
\Gamma_{2} \ll \Gamma_{1}, \quad \gamma_{1} \ll \gamma_{2} .
$$

Эти требования обеспечивают преимущественное заселение верхнего лазерного уровня активной среды и нижнего уровня пассивной среды.

Для простоты также положим, что частоты переходов активной и пассивной сред $\omega_{a}$ и $\omega_{p}$ равны друг другу и совпадают с частотой продольной моды $\omega_{0}$. Это означает, что мы должны положить $\delta_{a}=0$ и $\delta_{p}=0$. Нужно отметить, что, вообще говоря, частота лазерной генерации будет отличаться от частоты $\omega_{0}$, если иметь в виду генерацию солитона.

Поскольку время развития атомных переменных, согласно (70), значительно короче времени развития лазерного поля, то мы можем указать такой временной интервал, на котором атомная среда успевает перейти в стационарное состояние, а поле практически останется неизменным. По прошествии этого интервала имеем право в уравнениях (64)-(69) положить равными нулю все производные по времени. В результате получим равенства для активной среды:

$$
\begin{gathered}
\Gamma \sigma=g\left(\sigma_{2}-\sigma_{1}\right) a+F, \\
\Gamma_{1} \sigma_{1}=g\left(\sigma^{*} a+\sigma a^{*}\right)+F_{1}, \\
\Gamma_{2} \sigma_{2}=R_{a}-g\left(\sigma^{*} a+a^{*} \sigma\right)+F_{2}
\end{gathered}
$$

и для пассивной среды:

$$
\begin{gathered}
\gamma \pi=g_{p}\left(\pi_{2}-\pi_{1}\right) a+G, \\
\gamma_{1} \pi_{1}=R_{p}+g_{p}\left(\pi^{*} a+a^{*} \pi\right)+G_{1}, \\
\gamma_{2} \pi_{2}=-g_{p}\left(\pi^{*} a+\pi a^{*}\right)+G_{2} .
\end{gathered}
$$

Усредняя эти равенства, получим систему алгебраических уравнений для средних амплитуд $\bar{\sigma}, \bar{\sigma}_{1}, \bar{\sigma}_{2}, \bar{\pi}, \bar{\pi}_{1}$, $\bar{\pi}$. Здесь мы положили, что усреднение не затрагивает амплитуду лазерной генерации $a$ в силу ее медленного развития. Решая полученные алгебраические уравнения, сможем записать полуклассические стационарные заселенности актуальных атомных уровней и стационарные когерентности между этими уровнями:

$$
\begin{gathered}
\bar{\sigma}_{1}=\frac{R_{a}}{\Gamma_{1}} \frac{I}{1+I}, \quad \bar{\sigma}_{2}=\frac{R_{a}}{\Gamma_{2}} \frac{1}{1+I}, \\
g \bar{\sigma}=\frac{1}{2} \frac{A a}{1+I}, \\
\bar{\pi}_{1}=\frac{R_{p}}{\gamma_{1}} \frac{1}{1+I_{p}}, \quad \bar{\pi}_{2}=\frac{R_{p}}{\gamma_{2}} \frac{I_{p}}{1+I_{p}}, \\
g_{p} \bar{\pi}=-\frac{1}{2} \frac{A_{p} a}{1+I_{p}} .
\end{gathered}
$$

Здесь $I=\beta|a|^{2}-$ безразмерная мощность лазерной генерации и $I_{p}=v I$, где $v=\beta_{p} / \beta$. Величины $A=\beta R_{a}$ и $A_{p}=\beta_{p} R_{p}$ - линейные коэффициенты усиления активной среды и поглощения пассивной среды. Здесь обозначено

$$
\beta=\frac{4 g^{2}}{\Gamma_{1} \Gamma_{2}}, \quad \beta_{p}=\frac{4 g_{p}^{2}}{\gamma_{1} \gamma_{2}} .
$$

Величины $\beta^{-1}, \beta_{p}^{-1}$ имеют смысл числа фотонов, насыщающих соответствующий переход.

Стационарные корреляционные соотношения для ланжевеновских источников приобретают следующий вид (см. Приложение В) для активной среды:

$$
\begin{gathered}
\left\langle|F|^{2}\right\rangle=\Gamma_{1} \bar{\sigma}_{2}+R_{a}, \\
\left\langle F^{2}\right\rangle=2 g \bar{\sigma} a, \\
\left\langle F_{1} F\right\rangle=\Gamma_{1} \bar{\sigma}, \\
\left\langle F_{2}^{2}\right\rangle=2 R_{a}\left(1-s_{a} / 2\right)-2 \Gamma_{1} \bar{\sigma}_{1}, \\
\left\langle F_{1}^{2}\right\rangle=0, \\
\left\langle F_{2} F_{1}\right\rangle=\Gamma_{1} \bar{\sigma}_{1}
\end{gathered}
$$

и для пассивной среды:

$$
\begin{gathered}
\left\langle|G|^{2}\right\rangle=\gamma_{1} \bar{\pi}_{2}, \\
\left\langle G^{2}\right\rangle=2 g_{p} \bar{\pi} a, \\
\left\langle G_{1} G\right\rangle=\gamma_{1} \bar{\pi}, \\
\left\langle G_{1}^{2}\right\rangle=2 R_{p}\left(1-s_{p} / 2\right), \\
\left\langle G_{2}^{2}\right\rangle=2 \gamma_{2} \bar{\pi}_{2}, \\
\left\langle G_{1} G_{2}\right\rangle=-\gamma_{2} \bar{\pi}_{2} .
\end{gathered}
$$

Здесь учтено, что из формул (72)-(77) следуют полезные равенства для активной:

$$
\begin{gathered}
g\left(a^{*} \bar{\sigma}+a \bar{\sigma}^{*}\right)=\Gamma_{1} \bar{\sigma}_{1}, \\
\Gamma_{1} \bar{\sigma}_{1}+\Gamma_{2} \bar{\sigma}_{2}=R_{a}
\end{gathered}
$$


и пассивной сред:

$$
\begin{gathered}
g_{p}\left(a^{*} \bar{\pi}+a \bar{\pi}^{*}\right)=-\gamma_{2} \bar{\pi}_{2}, \\
\gamma_{1} \bar{\pi}_{1}+\gamma_{2} \bar{\pi}_{2}=R_{p} .
\end{gathered}
$$

Алгебраические равенства (72)-(77) дают возможность вычислить в явном виде величины

$$
g \sigma=g \bar{\sigma}+\Phi, \quad g_{p} \pi=g_{p} \bar{\pi}+\Phi_{p} .
$$

Эти выражения теперь следует подставить в уравнение для полевой амплитуды (63). В результате этой операции получим следующее замкнутое уравнение:

$$
\begin{aligned}
\frac{\partial a}{\partial t} & -\frac{i c}{2 k_{0}} \Delta_{\perp} a=-\frac{1}{2} \kappa a \\
& +\frac{1}{2}\left(\frac{A}{1+\beta|a|^{2}}-\frac{A_{p}}{1+\beta_{p}|a|^{2}}\right) a+\Phi+\Phi_{p},
\end{aligned}
$$

Это уравнение уже может быть базовым для достижения наших целей. Оно способно в полной мере описать генерацию солитона. Имеется, однако, еще одно обстоятельство, которое важно иметь в виду, если мы хотим следить за статистическими свойствами солитона. Речь идет о том, что рассматриваемое уравнение дает решение с полностью неопределенной фазой электромагнитного поля, что, вообще говоря, препятствует возникновению квантовых особенностей в поле. Например, строго говоря, квадратурное сжатие оказывается невозможным. Чтобы подавить фазовую диффузию, необходимо ввести в рассмотрение какой-нибудь синхронизирующий фактор. Внешнее поле в когерентном состоянии, вводимое извне в световой луч, вполне обеспечивает эту функцию.

Будем полагать, что синхронизирующее поле вводится через зеркало лазерного резонатора на частоте $\omega_{0}+v_{\text {in }}$, отличной, вообще говоря, от частоты продольной моды $\omega_{0}$. Тогда уравнение (96) перепишется в виде

$$
\begin{aligned}
\frac{\partial a}{\partial t} & -\frac{i c}{2 k_{0}} \Delta_{\perp} a=-\frac{1}{2} \kappa\left(a-a_{\text {in }} e^{-i v_{\text {in }} t}\right) \\
& +\frac{1}{2}\left(\frac{A}{1+\beta|a|^{2}}-\frac{A_{p}}{1+\beta_{p}|a|^{2}}\right) a+\Phi+\Phi_{p} .
\end{aligned}
$$

Здесь $a_{\text {in }}=\sqrt{n_{\text {in }}} \exp \left(i \varphi_{\text {in }}\right)-$ амплитуда синхронизирующего поля, пересчитанная на внутрирезонаторное пространство.

В выражениях (95)-(97) буквами $\Phi$ и $\Phi_{p}$ обозначены ланжевеновские источники, возникшие из исходных источников вследствие осуществления процедуры адиабатического исключения атомных переменных.

\section{6. Ланжевеновские источники в адиабатической $c$-числовой теории}

В адиабатическом уравнении (97) ланжевеновская сила представлена двумя слагаемыми $\Phi$ и $\Phi_{p}$. Первое из них формируется активной средой и оказывается комбинацией исходных источников $F, F_{1}, F_{2}$, а второе является комбинаций источников $G, G_{1}, G_{2}$, т.е. формируется пассивной средой. Оказывается удобным для вычислений записать источники в виде

$$
\begin{gathered}
\Phi=\frac{1}{2} \frac{\beta a}{1+\beta|a|^{2}} \xi_{2}+\xi_{a}, \\
\Phi_{p}=-\frac{1}{2} \frac{\beta_{p} a}{1+\beta_{p}|a|^{2}} \xi_{p 1}+\xi_{p a},
\end{gathered}
$$

Здесь параметры $\xi_{a}, \xi_{2}$ определяют вклады от исходных источников $F, F_{1}, F_{2}$ в виде

$$
\begin{gathered}
\xi_{a}=-\frac{2 g^{2} a}{\Gamma_{1}^{2}} F_{1}+\frac{2 g}{\Gamma_{1}} F, \\
\xi_{2}=-\frac{2 g}{\Gamma_{1}}\left(a F^{*}+a^{*} F\right)+\frac{4 g^{2}}{\Gamma_{1}^{2}}|a|^{2} F_{1}+F_{2} .
\end{gathered}
$$

Соответственно параметры $\xi_{p a}, \xi_{p 1}$ определяют вклады от источников $G, G_{1}, G_{2}$ :

$$
\begin{gathered}
\xi_{p a}=\frac{2 g_{p}^{2} a}{\gamma_{2}^{2}} G_{2}+\frac{2 g_{p}}{\gamma_{2}} G, \\
\xi_{p 1}=\frac{2 g_{p}}{\gamma_{2}}\left(a G^{*}+a^{*} G\right)+\frac{4 g_{p}^{2}}{\gamma_{2}^{2}}|a|^{2} G_{2}+G_{1} .
\end{gathered}
$$

У нас имеются все возможности, чтобы рассчитать нужные средние. Нетрудно получить следующие ненулевые моменты:

$$
\begin{gathered}
\left\langle\xi_{i}^{*}(t, \boldsymbol{\rho}) \xi_{j}\left(t^{\prime}, \boldsymbol{\rho}^{\prime}\right)\right\rangle=\left\langle\xi_{i}^{*} \xi_{j}\right\rangle \delta\left(t-t^{\prime}\right) \delta^{2}\left(\boldsymbol{\rho}-\boldsymbol{\rho}^{\prime}\right), \\
\left\langle\xi_{2} \xi_{2}\right\rangle=2 R_{a}\left(1-s_{a} / 2\right), \quad\left\langle\xi_{p 1} \xi_{p 1}\right\rangle=2 R_{p}\left(1-s_{p} / 2\right), \\
\left\langle\xi_{a}^{*} \xi_{a}\right\rangle=\frac{\beta R_{a}}{1+\beta|a|^{2}}, \quad\left\langle\xi_{2} \xi_{a}\right\rangle=-\frac{\beta R_{a} a}{1+\beta|a|^{2}} .
\end{gathered}
$$

В выражении (103) индексы $i$ и $j$ принимают значения $\{a, 2, p a, p 1\}$. При вычислении средних существенным обстоятельством было то, что физические параметры $\left(\gamma_{1} / \gamma_{2} I_{p}\right),\left(\gamma_{1} / \gamma_{2}\right),\left(\Gamma_{2} / \Gamma_{1}\right)$ и $\left(\Gamma_{2} / \Gamma_{1} I\right)$ выбирались предельно малыми. При этом сохраняется возможность режима насыщения $\left(I, I_{p} \gg 1\right)$.

Теперь можем записать корреляционные соотношения для источников $\Phi$ и $\Phi_{p}$ :

$$
\begin{gathered}
\left\langle\Phi_{i}^{*}(t, \boldsymbol{\rho}) \Phi_{j}\left(t^{\prime}, \boldsymbol{\rho}^{\prime}\right)\right\rangle=\left\langle\Phi_{i}^{*} \Phi_{j}\right\rangle \delta\left(t-t^{\prime}\right) \delta^{2}\left(\boldsymbol{\rho}-\boldsymbol{\rho}^{\prime}\right) \\
\left\langle\Phi^{2}\right\rangle=-\frac{1}{2}\left(\frac{\beta a}{1+I}\right)^{2} R_{a}\left(1+s_{a} / 2\right) \\
\left\langle|\Phi|^{2}\right\rangle=\frac{\beta}{1+I} R_{a}-\frac{1}{2}\left(\frac{\beta|a|}{1+I}\right)^{2} R_{a}\left(1+s_{a} / 2\right) \\
\left\langle\Phi_{p}^{2}\right\rangle=\frac{1}{2}\left(\frac{\beta_{p} a}{1+I_{p}}\right)^{2} R_{p}\left(1-s_{p} / 2\right)
\end{gathered}
$$



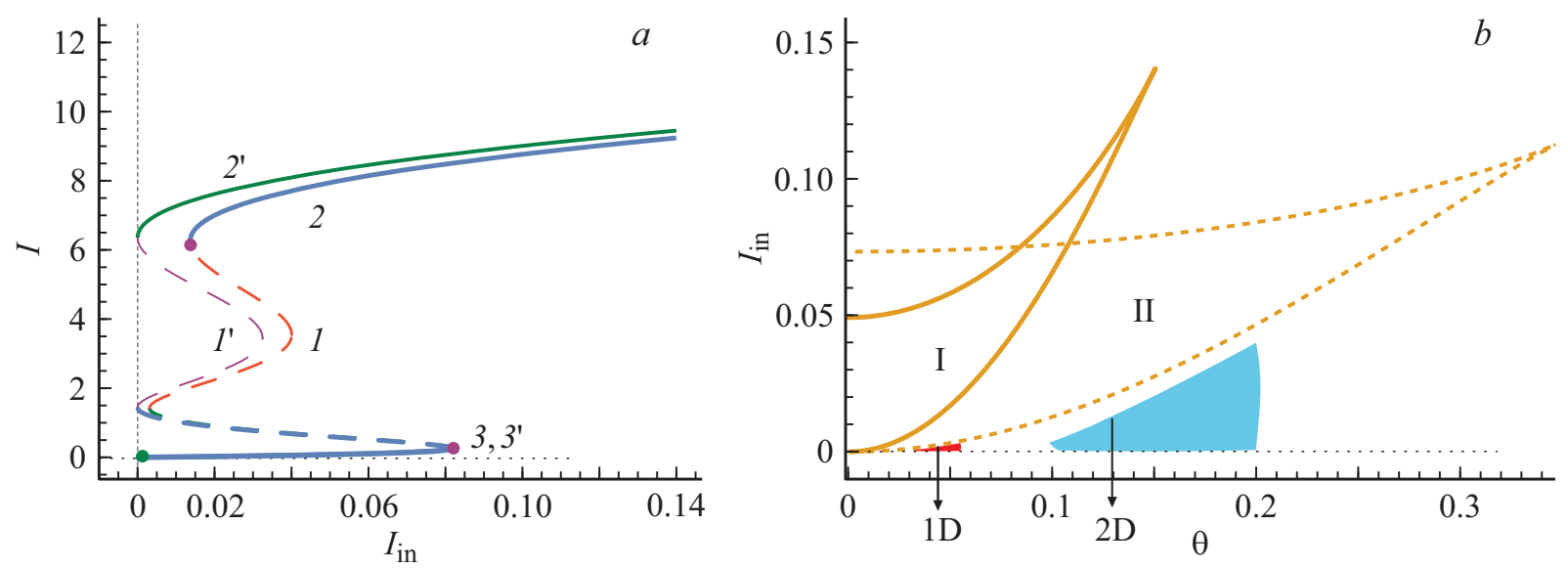

Рис. 2. (a) Гистерезисная зависимость интенсивности однородной генерации лазера от интенсивности поддерживающего излучения при коэффициенте усиления $g_{0}=2.08$. Кривые $1,1^{\prime}$ соответствует противофазному режиму, а кривые 2 и 3 - синфазному. Для кривых $1,2,3 \theta=0.047$, для $1^{\prime}, 2^{\prime}, 3^{\prime} \theta=0$. Штриховые и точечные линии обозначают неустойчивые, а непрерывные устойчивые решения. $(b)$ Области бистабильности синфазных (I) и противофазных (II) однородных режимов генерации и области захвата частоты одномерного (1D на графике) и двумерного (2D на графике) солитона, $g_{0}=2.08$ (1D), 2.11 (2D). Нижняя граница областей захвата соответствует интенсивности падающего пучка $I_{\text {in }} \approx 4 \cdot 10^{-6}$.

$$
\left\langle\left|\Phi_{p}\right|^{2}\right\rangle=\frac{1}{2}\left(\frac{\beta_{p}|a|}{1+I_{p}}\right)^{2} R_{p}\left(1-s_{p} / 2\right) .
$$

Здесь под индексами $i$ и $j$ в (105) имеется ввиду либо индекс $p$, либо отсутствие индекса. Таким образом, базовое уравнение для лазерного солитона, полученное в виде уравнения Гейзенберга-Ланжевена (97), оказывается полностью сформулированным. Нужно отметить, что выражения (108), (109) отличаются от тех, что были получены в работе [18]. Это является прямым следствием того, что здесь коллективные переменные мы ввели иначе.

\section{7. Классическая теория лазера с насыщающимся поглощением и внешним сигналом}

\section{1. Управляющее уравнение}

В дальнейшем мы предполагаем исследовать квантовые особенности солитона, проявляющиеся на фоне классического солитона. Поэтому в этом разделе опишем основные свойства классического солитона.

В пренебрежении квантовыми флуктуациями уравнение (97) записывается в следующем безразмерном виде, совпадающем при $E_{\text {in }}=0$ с принятым в наших предшествовавших работах, $[1,13]$ :

$$
\frac{\partial E}{\partial t}-i \Delta_{\perp} E=E_{\text {in }}+i \theta E+f\left(|E|^{2}\right) E .
$$

Здесь время нормировано на линейные амплитудные потери, поперечная координата - на ширину зоны Френеля, интенсивность - на интенсивность насыщения поглотителя, $b=\beta_{p} / \beta-$ отношение интенсивностей насыщения активных и пассивных центров, $\nabla_{\perp}^{2}=\nabla_{\boldsymbol{\rho}}^{2}$ (штрихи в (101) опущены):

$$
\begin{gathered}
t=t^{\prime} /(\kappa / 2), \quad \boldsymbol{\rho}=\boldsymbol{\rho}^{\prime} / \sqrt{c /\left(\kappa k_{0}\right)}, \\
a(t, \boldsymbol{\rho})=E\left(t^{\prime}, \boldsymbol{\rho}^{\prime}\right) e^{-i v_{\text {in }} t} / \sqrt{\beta_{p}} .
\end{gathered}
$$

Для безразмерных коэффициентов усиления и поглощения в центре линий $g_{0}$ и $a_{0}$ и полного нелинейного коэффициента усиления $f(I)$ используем обозначения $[1,13]$, которые отвечают условию $E_{\text {in }}=0$ :

$$
\begin{gathered}
g_{0}=A / \kappa, \quad a_{0}=A_{p} / \kappa, \\
f(I)=-1-\frac{a_{0}}{1+I}+\frac{g_{0}}{1+I / b}, \quad I=|E|^{2} .
\end{gathered}
$$

Здесь было удобней считать частоту внешнего сигнала (поддерживающего излучения) несущей частотой и ввести частотную расстройку между ней и частотой продольной моды резонатора $\theta=\left(v_{\text {in }}-v_{0}\right) /(\kappa / 2)$. Как и выше, частота этой моды отвечает центрам линий усиления и поглощения. Вообще говоря, при $\theta \neq 0$ функция $f(I)$ становится комплексной. Но если частотный сдвиг много меньше ширины линии, то мнимая часть $f$ мала, и ею можно пренебречь. В численных расчетах используются фиксированные значения параметров $b=10, a_{0}=2, g_{0}=2.08$ для поперечно-одномерной и $g_{0}=2.11$ для двумерной геометрии.

\section{2. Стационарная однородная генерация}

Рассмотрим сначала пространственно однородные стационарные решения (110), считая без ограничения общности $E_{\text {in }}>0$. Для них, полагая $E=\sqrt{I} e^{i \varphi}$, сводим уравнение (110) к виду

$$
e^{-i \varphi} E_{\text {in }} / \sqrt{I}=-i \theta-f(I) .
$$



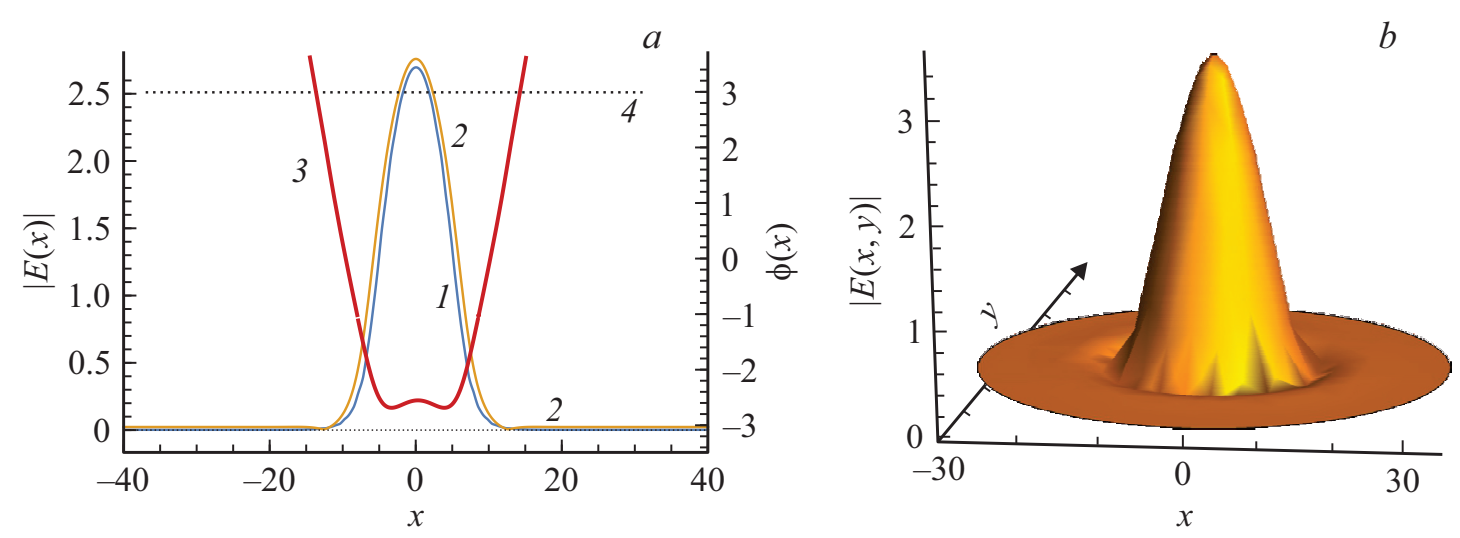

Рис. 3. Профили амплитуды для одномерного $(a)$ и двумерного $(b)$ синхронизированных солитонов. Кривая $1-$ установившийся лазерный солитон без поддерживающего пучка, $2-$ синхронизированный солитон для $E_{\text {in }}=0.02, \theta=0.043,3-$ профиль фазы синхронизированного солитона, $4-$ амплитуда однородного режима генерации. $g_{0}=2.08(a), 2.11(b)$.

Отсюда видно, что при точном резонансе $(\theta=0)$ существуют два решения: синфазное, $\varphi=0$, и противофазное, $\varphi=\pi$. На рис. 2, $a$ приведена многозначная зависимость интенсивности однородного режима $I$ от интенсивности поддерживающего излучения $I_{\text {in }}=\left|E_{\text {in }}\right|^{2}$ при постоянном значении $g_{0}$ для двух значений расстроек $\theta$. Устойчивость решений (115) проверяется вычислением инкремента роста малых возмущений в рамках линеаризованных уравнений (110). Для выбранных параметров нижняя и верхняя ветви этих кривых в синфазном режиме отвечают устойчивым режимам, а все противофазные режимы неустойчивы.

\section{3. Режим синхронизации солитона}

В лазере с насыщающимся поглотителем без поддерживающего излучения существуют различные типы пространственных солитонов, отвечающие поперечно локализованной генерации с определенной частотой $v_{s}[1,13]$. Последняя является собственным значением задачи

$$
\Delta_{\perp} A+\left(v_{s}+\theta\right)=i f\left(|A|^{2}\right) A,
$$

возникающей при подстановке $E=A(\boldsymbol{\rho}) \exp \left(-i v_{s} t\right)$ в управляющее уравнение (110). При весьма слабом поддерживающем излучении поле внутри резонатора можно рассматривать как суперпозицию поля солитона и поля, отвечающего устойчивой однородной генерации с частотой поддерживающего излучения. При несовпадении этой частоты с частотой солитона общее поле слабо промодулировано по времени с разностной частотой $v_{s}-v_{\text {in. }}$ Поэтому для синхронизации солитона поддерживающим излучением требуется, чтобы интенсивность поддерживающего излучения превосходила некоторое критическое значение, зависящее от частотной расстройки $\theta$. На рис. 2, $b$ показаны области параметров, в которых достигается такая синхронизация в поперечно одномерной и двумерной геометриях резонатора. Из рисунка видно, что эти области находятся вне областей бистабильности однородных режимов генерации. Расчеты показывают, что не только синхронизация, но и локализация структуры исчезает при превышении интенсивностью поддерживающего излучения некоторого другого критического значения. При этом происходит последовательное расширение области либо низкоинтенсивной однородной генерации на всю апертуру лазера, либо генерация с биениями в зависимости от частотной расстройки. Отметим, что при оптимальном выборе частотной расстройки для достижения синхронизации достаточно поддерживающее излучение с весьма малой интенсивностью $I_{\text {in }} \approx 4 \cdot 10^{-6}$. Поперечный профиль синхронизованного солитона приведен на рис. 3 также для двух вариантов геометрии. На периферии солитона поле стремится к полю однородной генерации, рассмотренной выше. Соответствующий фон имеет весьма малую величину $(|E| \approx 0.02)$. При этом в периферийной части наблюдаются пространственные биения, которые можно интерпретировать как результат интерференции полей собственно солитона и поля однородной генерации. В центральной части солитона существенных изменений профиля солитона по сравнению с вариантом отсутствия поддерживающего излучения не происходит.

Наряду с балансом усиления и поглощения существенным фактором для устойчивости локализованной структуры является совпадение частоты внешнего излучения с частотой лазерного солитона без внешнего пучка, которая задается нелинейным сдвигом частоты относительно продольной моды резонатора $v$. На рис. 3 представлен профиль поля для случая полной синхронизации, когда безразмерная расстройка $\theta=v /(\kappa / 2)$. Рисунок 4 демонстрирует кинетику установления амплитуды, фазы и частоты в максимуме одномерного солитона для двух случаев: без поддерживающего излучения и с внешним, полностью синхронизирующим пучком. В первом случае устанавливается ненулевое значение частоты и линейное спадание фазы, во втором случае 

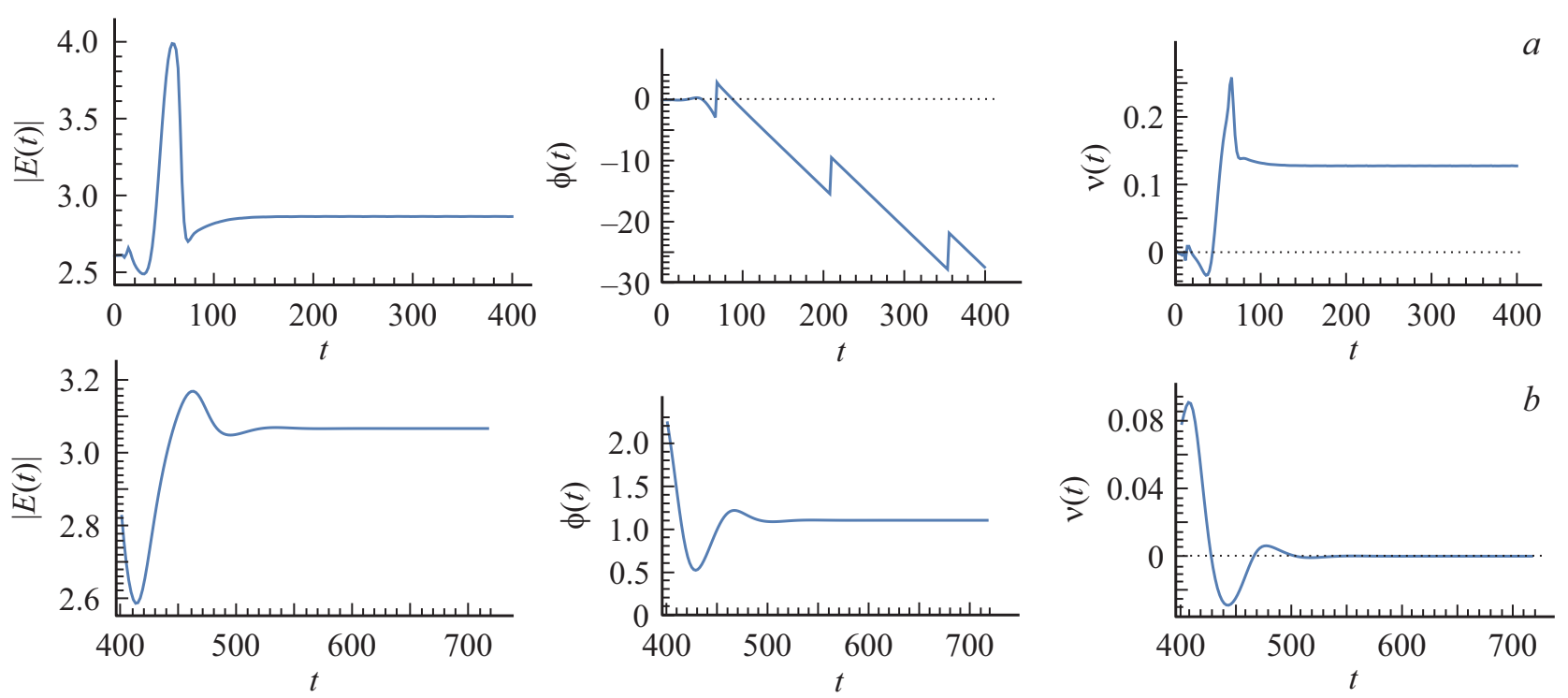

Рис. 4. Кинетика установления амплитуды, фазы и частоты в 2D-солитоне. $(a)$ Лазер без поддерживающего пучка, $(b)$ Cинхронизация с частотой поддерживающего пучка. Параметры $E_{\text {in }}=0(a), 0.04(b), \theta=0.129(b)$. Линейное снижение фазы на рисунке сопровождается скачками на $2 \pi$.
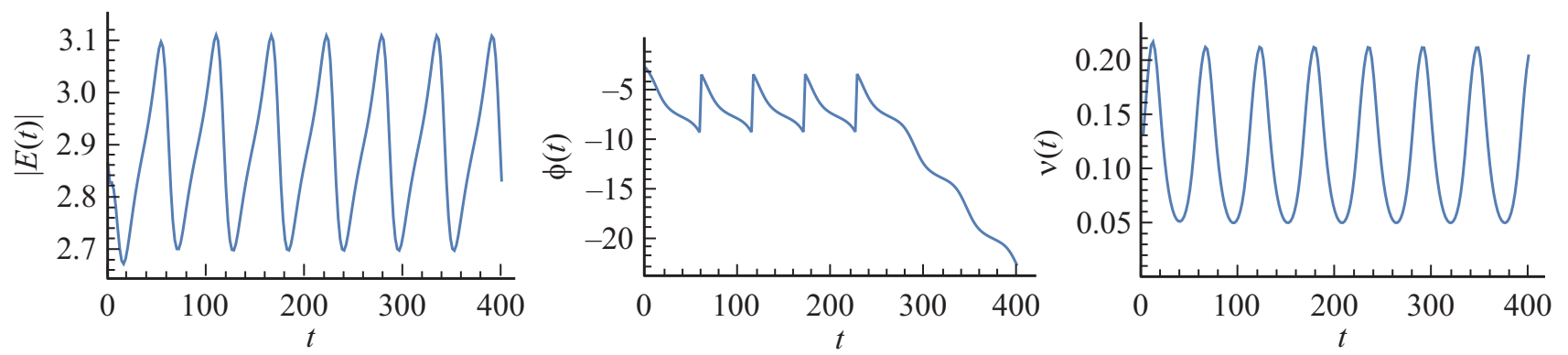

Рис. 5. Кинетика биений амплитуды, фазы и частоты в $2 \mathrm{D}$-солитоне. Параметры $E_{\text {in }}=0.04, \theta=0$.
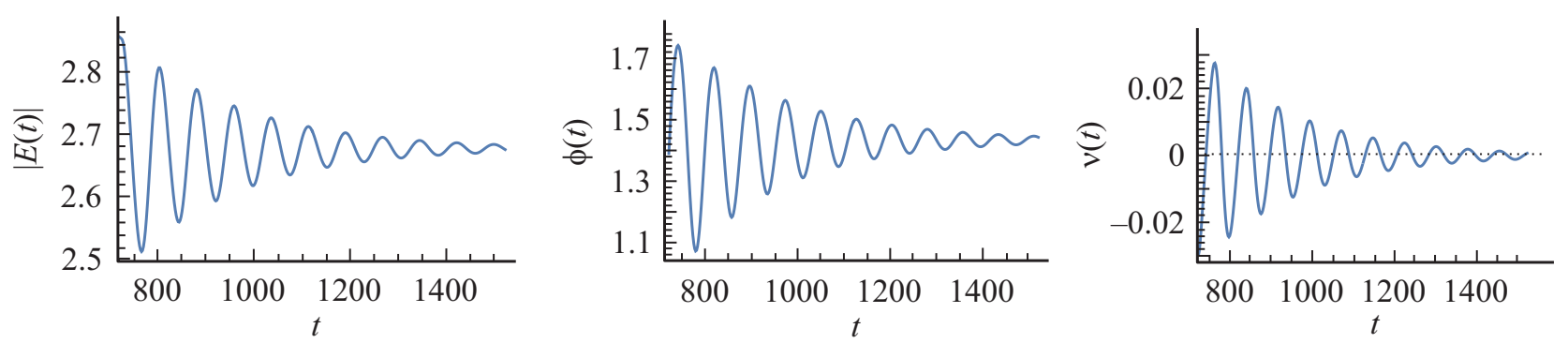

Рис. 6. Кинетика медленного установления амплитуды, фазы и частоты в 2D-солитоне вблизи границы области захвата частоты. Параметры $E_{\text {in }}=0.04, \theta=0.2$.

происходит синхронизация, сдвиг частоты солитона относительно внешнего пучка стремится к нулю, а сдвиг фазы становится постоянным.

Учет нелинейного сдвига частоты лазерного солитона существен для синхронизации его с внешним пучком излучения. Если частота внешнего пучка совпадает с частотой продольной моды, а амплитуда не превышает критического значения, солитон не разваливается, но осциллирует с частотой нелинейного сдвига. На рис. 5 показана кинетика биений лазерного солитона в этом случае.

При увеличении расстройки выше значения нелинейного сдвига также происходит переход от состояния синхронизации к биениям солитона. На рис. 6 приведен пример кинетики установления режима синхронизации вблизи границы области захвата частоты, но внутри этой области. Видно, что фаза поля устанавливается медленно, но амплитуда биений постепенно уменьша- 
ется до нуля. Области захвата частоты в одномерном и двумерном солитонах представлены на рис. 2, $b$. Уменьшение амплитуды падающего пучка в области захвата приводит к появлению биений с небольшой амплитудой, причем частота биений увеличивается при увеличении частотной расстройки падающего пучка с собственной частотой солитона. При точном совпадении этих частот период биений стремится к бесконечности, т.е. при $E_{\text {in }} \rightarrow 0$ интервал расстроек захвата частоты сужается до нуля вблизи нелинейного сдвига частоты.

\section{Заключение}

Представленная работа является одной из серии работ, выполняемых нами по изучению динамических и квантово-статистических свойств лазерного солитона. Первая публикация [18] рассматривалась как предварительная и была посвящена лазерной генерации с участием в процессе насыщенного поглощения. В этом случае никакие пространственные проявления не учитывались, т.е. напрямую к проблеме лазерного солитона это рассмотрение отношения не имело. Тем не менее это позволило нам оценить важные методические особенности генерации лазера в присутствии поглощающей среды на предмет их дальнейшего использования для анализа солитона.

На этой методической базе в настоящей работе было выведено нелинейное уравнение Гейзенберга-Ланжевена в частных производных, описывающее формирование солитона и его статистику. При выводе предполагалось, что выбранная для обсуждения система может быть описана в рамках адиабатического приближения. Это достигается, как известно, требованием медленного развития лазерного поля по сравнению с развитием обеих (активной и пассивной) атомных сред.

Тем самым на этом этапе мы подготовили возможность для всестороннего описания системы. Все вопросы, связанные с квантово-статистическим описанием, могут быть выяснены при решении полученного основного уравнения. В этой работе мы провели численный анализ решения уравнения в классическом пределе. Отметим, этот этап оказывается важным не только сам по себе, но и для статистического описания, так как мы следим за квантовыми флуктуациями на фоне именно классического солитона.

Полученное уравнение дает возможность исследовать лазерный солитон на наличие или отсутствие в нем квантовых особенностей. Для того, чтобы обеспечить достоверность результатов в квантовой теории, мы должны очень аккуратно конструировать ланжевеновские источники. Сначала следует построить все ланжевеновские источники на базовом уровне. При этом каждой канонической переменной будет соответствовать свой собственный источник, и каждый из этих источников в адиабатическом приближении так или иначе формирует искомый источник в уравнении для поля генерации.

Что касается квантовых особенностей, они могут возникать по двум причинам. Во-первых, вследствие внешних причин, а именно из-за субпуассоновского характера возбуждения активной (или даже пассивной) среды. Во-вторых, вследствие внутренних причин, связанных со сложным нелинейным взаимодействием поля и вещества. В дальнейшем мы предполагаем прояснить эти вопросы на основе полученного в этой статье уравнения.

\section{Финансирование работы}

Работа поддержана Российским фондом фундаментальных исследований, грант 18-02-00402а. Изучение возможности синхронизации классического солитона выполнено за счет гранта Российского научного фонда (проект № 18-12-00075).

\section{Конфликт интересов}

Авторы заявляют, что у них нет конфликта интересов

\section{Приложение А. Субпуассоновская лазерная генерация с насыщающим поглощением}

\section{1. Введение}

Полученное в основном тексте уравнение (97) оказывается пригодным для обсуждения довольно широкого круга физических условий. Во-первых, это лазерные задачи о влиянии насыщенного поглощения на генерацию. При этом возможно учитывать пространственный аспект или не учитывать. Во-вторых, это задачи по генерации лазерного солитона с внешней вынуждающей силой, синхронизирующей поле в резонаторе или без нее. Все эти задачи методически близки друг к другу, поэтому имеет смысл их обсуждать по возможности совместно.

Наиболее простая из них это лазерная задача в приближении плоской волны при наличии в системе насыщенного поглощения. Ее мы уже обсудили в работе [18]. Там были рассмотрены две возможные измерительные процедуры, связанные с наблюдением спектра флуктуаций числа фотонов в лазерной генерации и с наблюдением квадратурного сжатия. При этом предполагалось, что возбуждение лазерной активной среды может быть как совершенно случайным пуассоновским процессом, так и регулярным субпуассоновским.

Решение задачи о лазере с насыщенным поглощением имеет двоякий смысл. Во-первых, это сама по себе интересная физическая ситуация. Во-вторых, в методическом плане она существенно пересекается с задачей по генерации лазерного солитона и потому может быть полезной чисто технически, поскольку лазерная система 
в формальном отношении заметно проще солитонной хотя бы потому, что последняя содержит в себе пространственный аспект. В настоящий момент, однако, возникла ситуация, затрудняющая проводить потенциально полезные сравнительные процедуры между лазерной задачей [18] и задачей, решаемой здесь, и учитывающей пространственный аспект.

Дело в том, что и там, и здесь задачи решались в терминах коллективных переменных для обеих, активной и пассивной, сред. При этом здесь мы вводим оператор коллективных когерентностей для обеих сред одинаковым способом, а в работе [18] - по-разному. Это привело к тому, что сравнительные процедуры оказались затруднительными. Это, разумеется, неудобно, и мы в этом Приложении постараемся скомпенсировать это неудобство, переформулировав определение коллективных когерентностей.

\section{2. Основное уравнение}

\section{для лазерной генерации}

Будем решать задачу в приближении плоской волны, т.е. полностью игнорируем пространственный аспект. Полагая в уравнении (97) $v_{\text {in }}=0$, перепишем его в виде

$$
\begin{aligned}
\dot{a}= & -\frac{1}{2} \kappa\left(a-a_{\text {in }}\right) \\
& +\frac{1}{2}\left(\frac{A}{1+\beta|a|^{2}}-\frac{A_{p}}{1+\beta_{p}|a|^{2}}\right) a+\Phi+\Phi_{p} .
\end{aligned}
$$

Все другие частотные отстройки тоже равны нулю: $\omega_{0}=\omega_{a}=\omega_{p}$.

Напоминаем, что это уравнение является следствием операторного уравнения

$$
\dot{\hat{a}}=-\frac{1}{2} \kappa\left(\hat{a}-\hat{a}_{\text {in }}\right)+g \hat{\sigma}+g_{p} \hat{\pi}+\hat{F}_{a} .
$$

Здесь $\hat{\sigma}$ и $\hat{\pi}-$ коллективные когерентности активной и пассивной сред. Эти переменные в данной работе определены как линейные суперпозиции индивидуальных когерентностей $\hat{\sigma}^{j}$ и $\hat{\pi}^{j}$ :

$$
\begin{array}{ll}
\hat{\sigma}=-i \sum_{j} \hat{\sigma}^{j} \Theta\left(t-t_{j}\right), & \hat{\sigma}^{j}=|1\rangle_{j j}\langle 2|, \\
\hat{\pi}=-i \sum_{j} \hat{\pi}^{j} \Theta\left(t-t_{j}\right), & \hat{\pi}^{j}=|1\rangle_{j j}\langle 2| .
\end{array}
$$

В работе [18] оператор $\hat{\pi}$ определен несколько иначе, a именно он представлен как линейная суперпозиция индивидуальных операторов $\hat{\pi}^{j \dagger}$.

Что касается ланжевеновских источников, то в согласии с выражениями (105)-(109) для активной среды можем написать следующие ненулевые корреляционные соотношения:

$$
\begin{gathered}
\left\langle\Phi^{2}\right\rangle=-\frac{1}{2}\left(\frac{\beta a}{1+I}\right)^{2} R_{a}\left(1+s_{a} / 2\right), \\
\left\langle|\Phi|^{2}\right\rangle=\frac{\beta}{1+I} R_{a}-\frac{1}{2}\left(\frac{\beta|a|}{1+I}\right)^{2} R_{a}\left(1+s_{a} / 2\right) .
\end{gathered}
$$

Для пассивной среды имеем

$$
\begin{array}{r}
\left\langle\Phi_{p}^{2}\right\rangle=\frac{1}{2}\left(\frac{\beta_{p} a}{1+I_{p}}\right)^{2} R_{p}\left(1-s_{p} / 2\right), \\
\left\langle\left|\Phi_{p}\right|^{2}\right\rangle=\frac{1}{2}\left(\frac{\beta_{p}|a|}{1+I_{p}}\right)^{2} R_{p}\left(1-s_{p} / 2\right) .
\end{array}
$$

Заметим, что последние две формулы в работе [18] выглядят иначе. Это прямое следствие того, что мы иначе определили коллективную когерентность пассивной среды.

\section{3. Флуктуации числа фотонов}

Сначала положим, что внешнее синхронизирующее поле отсутствует $\left(a_{\text {in }}=0\right)$ и запишем амплитуду поля в виде $a=\sqrt{n} \exp (i \varphi)$. Тогда вместо исходного уравнения (117) получим два уравнения вида

$$
\begin{gathered}
\dot{n}=-\kappa n+\left(\frac{A}{1+\beta n}-\frac{A_{p}}{1+\beta_{p} n}\right) n+\Phi_{n}, \\
\dot{\varphi}=\Phi_{\varphi} .
\end{gathered}
$$

Здесь ланжевеновские источники выражаются через исходные следующим образом:

$$
\begin{array}{r}
\Phi_{n}=\left[a^{*} \Phi+a \Phi^{*}\right]+\left[a^{*} \Phi_{p}+a \Phi_{p}^{*}\right], \\
\Phi_{\varphi}=-\frac{i}{n}\left[a^{*} \Phi-a \Phi^{*}\right]-\frac{i}{n}\left[a^{*} \Phi_{p}-a \Phi_{p}^{*}\right] .
\end{array}
$$

Будем искать решение уравнения (125) в виде

$$
n(t)=\bar{n}+\varepsilon(t), \quad \bar{n} \gg \varepsilon(t) .
$$

Здесь $\bar{n}-$ стационарное классическое решение, для которого имеет место равенство

$$
\kappa=\frac{A}{1+\beta \bar{n}}-\frac{A_{p}}{1+\beta_{p} \bar{n}} .
$$

Проведя процедуру линеаризации уравнения (125) по флуктуации $\varepsilon$, получаем простое уравнение вида

$$
\dot{\varepsilon}=-D \varepsilon+\Phi_{n},
$$

где коэффициент $D$ выражен через лазерные параметры с учетом равенства (130) следующим образом:

$$
D=\kappa \frac{I}{1+I}\left[1+\frac{\left(A_{p} / \kappa\right)(1-v)}{(1+v I)^{2}}\right], \quad v=\beta_{p} / \beta .
$$


Как видно, при $v<1$ величина $D$ всегда положительна, т. е. стационарное решение задачи всегда устойчиво. В то же время при $v>1$ стационарное решение оказывается устойчивым только для не слишком большой величины $v<v_{0}$, где

$$
v_{0}=\frac{\kappa_{p} / \kappa+1}{\kappa_{p} / \kappa-I}, \quad \kappa_{p}=\frac{A_{p}}{1+v I} .
$$

Перепишем уравнение (131) в спектральном представлении:

$$
\varepsilon_{\omega}=\frac{\Phi_{n, \omega}}{D-i \omega} .
$$

Чтобы получить спектр ланжевеновского шума, примем во внимание определение (127), и тогда

$$
\left\langle\left|\Phi_{n, \omega}\right|^{2}\right\rangle=2 \tilde{n} D_{1}
$$

где

$$
\begin{aligned}
D_{1}= & \kappa \frac{1-\left(s_{a} / 2\right) I}{1+I} \\
& +\frac{A_{p}}{1+v I}\left(\frac{1-\left(s_{a} / 2\right) I}{1+I}+\frac{\left(1-s_{p} / 2\right) v I}{1+v I}\right) .
\end{aligned}
$$

Явное выражение для коэффициента $D_{1}$ в основном совпадает с тем, что было получено в работе [18]. Сделано только два уточнения. Во-первых, мы ради общности учли возможность регулярного возбуждения не только активной, но и пассивной сред. В результате в формуле появился дополнительный фактор $\left(0<s_{p}<1\right)$. Во-вторых, в прежней формуле в круглых скобках ошибочно исчез множитель $(v I)$, стоящий в числителе второго слагаемого.

Применяя стандартную измерительную процедуру, можем получить спектр шумов фототока в виде

$$
\left\langle\left|\delta i_{\omega}\right|^{2}\right\rangle /\langle i\rangle=1+\frac{2 \kappa D_{1}}{D^{2}+\omega^{2}}, \quad\langle i\rangle=\kappa|\tilde{a}|^{2} .
$$

Напоминаем, что эта формула верна только при выполнении условия устойчивости стационарной генерации (133).

\section{4. Фазовая диффузия в лазерном поле без синхронизации}

Теперь проследим за тем, что происходит с фазой поля генерации. Напоминаем, что согласно полуклассической теории фаза оказывается случайной величиной и остается таким образом никак неопределенной. Чтобы понять, как к этому относится квантовая теория, нужно вернуться к уравнению (123). Проинтегрируем его на временном интервале от $t_{1}$ до $t_{2}$, тогда получим равенство:

$$
\varphi\left(t_{2}\right)-\varphi\left(t_{1}\right)=\int_{t_{1}}^{t_{2}} d t \Phi_{\varphi} .
$$

Поскольку среднее значение ланжевеновского источника равно нулю, $\left\langle\Phi_{\varphi}\right\rangle=0$, то это означает, что среднее значение фазы генерации со временем остается неизменным, $\left\langle\varphi\left(t_{2}\right)\right\rangle=\left\langle\varphi\left(t_{1}\right)\right\rangle$. Более того, мы не можем предсказать результат измерения фазы. И если мы ее все-таки определили, то вследствие ее флуктуаций мы теряем эту информацию через некоторое характерное время. Действительно, среднеквадратичные флуктуации фазы определяются величиной

$$
\begin{aligned}
\left\langle\left[\varphi\left(t_{2}\right)-\varphi\left(t_{1}\right)\right]^{2}\right\rangle & =\iint_{t_{1}}^{t_{2}} d t d t^{\prime}\left\langle\Phi_{\varphi}(t) \Phi_{\varphi}\left(t^{\prime}\right)\right\rangle \\
& =\left\langle\Phi_{\varphi}^{2}\right\rangle\left(t_{2}-t_{1}\right) .
\end{aligned}
$$

Мы можем получить, согласно (126),

$$
\left\langle\Phi_{\varphi}^{2}\right\rangle=\frac{2 \kappa}{\bar{n}}\left(1+\frac{A_{p} / \kappa}{1+v I}\right) .
$$

Выражение (139) трактуем как фазовую диффузию, которая разрушает информацию о фазе за характерное время диффузии $\left\langle\Phi_{\varphi}^{2}\right\rangle^{-1}$. Как видим, насыщающая ячейка уменьшает время диффузии. Кроме того, можем заключить, что в этом случае, когда синхронизация лазерного поля отсутствует, диффузия фазы никак не зависит от статистики фотонов. Действительно, результат не зависит от статистических параметров возбуждения среды $s_{a}$ и $s_{p}$.

Сравнивая последнюю формулу с той, которая была получена в работе [18], видим, что здесь коэффициент 2 общий, а в работе [18] он относится только ко второму слагаемому в круглых скобках.

\section{5. Квадратурное сжатие лазерного поля с насыщенным поглощением в условиях фазовой синхронизации}

В предыдущем разделе мы обсуждали лазерную генерацию с насыщенным поглощением в отсутствие синхронизации. Там главными действующими переменными были флуктуации числа фотонов и флуктуации фазы. Там мы могли увидеть, что при регулярной накачке атомов активной среды статистика фотонов оказывалась субпуассоновской с полным или частичном подавлением дробовых флуктуаций в режиме насыщения при $I, v I \gg 1$. В то же время насыщенное поглощение могло заметно увеличить фазовую диффузию при условии $A_{p} / \kappa \gg 1$.

Чтобы понять роль синхронизации, возвратимся к уравнению (117). Если внешнее поле с амплитудой $a_{\text {in }}=\sqrt{n_{\text {in }}} \exp \left(i \varphi_{\text {in }}\right)$ находится в когерентном состоянии, то, как известно, фаза поля генерации „захватывается“ внешним полем. Флуктуации фазы при этом, разумеется, имеют место, но они оказываются теперь малыми. 
В связи с этим полагаем, что амплитуда поля генерации может быть записана в виде

$$
a(t)=\tilde{a}+\delta a(t), \quad \tilde{a} \gg \delta a(t) .
$$

Здесь стационарная амплитуда $\tilde{a}$ определяется равенством

$$
\begin{gathered}
\kappa(1-\mu)=\frac{A}{1+\beta|\tilde{a}|^{2}}-\frac{A_{p}}{1+\beta_{p}|\tilde{a}|^{2}}, \\
\mu=\sqrt{n_{\text {in }} / \tilde{n}} .
\end{gathered}
$$

Для нас наибольший интерес здесь представляют не сама комплексная амплитуда, а ее квадратуры. Флуктуации квадратур в удобном базисе записываются в виде

$$
\begin{aligned}
& \delta x=\frac{1}{2}\left(e^{-i \varphi_{\text {in }}} \delta a+e^{i \varphi_{\mathrm{in}}} \delta a^{*}\right), \\
& \delta y=\frac{1}{2 i}\left(e^{-i \varphi_{\text {in }}} \delta a-e^{i \varphi_{\text {in }}} \delta a^{*}\right) .
\end{aligned}
$$

Вследствие линеаризации уравнения (117) по флуктуациям квадратур получаем для этих квадратур два независимых уравнения:

$$
\begin{gathered}
\dot{\delta x}=-\left(\kappa \mu / 2+D_{\mu}\right) \delta x+\Phi_{x}, \\
\dot{\delta y}=-\kappa \mu / 2 \delta y+\Phi_{y} .
\end{gathered}
$$

Коэффициент $D_{\mu}$ теперь оказывается зависящим от параметра синхронизации $\mu$ и определяется выражением

$$
D_{\mu}=\kappa \frac{I}{1+I}\left[1-\mu+\frac{\left(A_{p} / \kappa\right)(1-v)}{(1+v I)^{2}}\right] .
$$

Здесь по сравнению с (132) появилась зависимость от $\mu$. Выражения полностью совпадают при $\mu=0$.

Что касается ланжевеновских источников в уравнениях (145), (146), они выражаются через исходные источники $\Phi$ и $\Phi_{p}$ следующим образом:

$$
\begin{aligned}
\Phi_{x}= & \frac{1}{2}\left[e^{-i \varphi_{\text {in }}} \Phi+e^{i \varphi_{\text {in }}} \Phi^{*}\right] \\
& +\frac{1}{2}\left[e^{-i \varphi_{\text {in }}} \Phi_{p}+e^{i \varphi_{\text {in }}} \Phi_{p}^{*}\right], \\
\Phi_{y}= & \frac{1}{2 i}\left[e^{-i \varphi_{\text {in }}} \Phi-e^{i \varphi_{\text {in }}} \Phi^{*}\right] \\
& +\frac{1}{2 i}\left[e^{-i \varphi_{\text {in }}} \Phi_{p}-e^{i \varphi_{\text {in }}} \Phi_{p}^{*}\right] .
\end{aligned}
$$

Далее будем обсуждать ситуацию на языке фурьекомпонент, тогда уравнения (145), (146) превратятся в равенства

$$
\begin{array}{r}
-i \omega \delta x_{\omega}=-\left(\kappa \mu / 2+D_{\mu}\right) \delta x_{\omega}+\Phi_{x \omega} \\
-i \omega \delta y_{\omega}=-\kappa \mu / 2 \delta y_{\omega}+\Phi_{y \omega}
\end{array}
$$

Теперь, принимая во внимание корреляционные соотношения для $\Phi$ и $\Phi_{p}(24)-(25)$, можем получить

$$
\begin{gathered}
\left\langle\left|\Phi_{x \omega}\right|^{2}\right\rangle=D_{1, \mu} / 2, \\
\left\langle\left|\Phi_{y \omega}\right|^{2}\right\rangle=\kappa / 2(1-\mu)+\frac{A_{p}}{1+\tilde{I}_{p}},
\end{gathered}
$$

где коэффициент $D_{1, \mu}$ имеет вид

$$
\begin{aligned}
D_{1, \mu}= & \kappa(1-\mu) \frac{1-\left(s_{a} / 2\right) I}{1+I}+\frac{A_{p}}{1+v I} \\
& \times\left(\frac{1-\left(s_{a} / 2\right) I}{1+I}+\frac{\left(1-\left(s_{p} / 2\right)\right) v I}{1+v I}\right) .
\end{aligned}
$$

Зная спектральные мощности шумов (36), (37), можно в явном виде записать спектральные мощности соответствующих квадратурных компонент внутри резонаторного поля, а используя хорошо известное input-output соотношение, связать их с наблюдаемыми снаружи резонатора.

Для наблюдения квадратур обычно применяется так называемое балансное гомодинное детектирование. В этом подходе сигнал с амплитудой $\hat{a}_{\text {out }}(t)$, вышедший из резонатора, прежде чем попасть на фотодетектор, смешивается с классическим полем локального осциллятора $L(t)$. Для нас наибольший интерес представляет именно $x$-квадратура поля. Имея в виду предыдущие формулы этого раздела, получим спектр шумов фототока в виде

$$
\left\langle\left|\delta i_{\omega}\right|^{2}\right\rangle /|L|^{2}=1+\frac{2 \kappa D_{1, \mu}}{\left(D_{\mu}+\kappa \mu / 2\right)^{2}+\omega^{2}} .
$$

В режиме насыщения обеих сред $D_{\mu}=\kappa(1-\mu)$, $D_{1, \mu}=-s_{a} / 2 \kappa(1-\mu)$. Нетрудно видеть, что при этом

$$
\left\langle\left|\delta i_{\omega=0}\right|^{2}\right\rangle /|L|^{2}=\mu^{2} / 4 \ll 1 .
$$

Мы можем сделать заключение, что дополнительная ячейка, введенная в лазерный резонатор, не приводит к разрушению квантовых особенностей субпуассоновского лазера независимо от того, сихронизован он внешним полем или нет.

\section{Приложение В. Ланжевеновские источники в уравнениях для двухуровневой среды}

В этой работе для расчетов были выбраны наиболее простые модели поля и вещества. Предположено, что лазерное поле - это квазиплоская и квазимонохроматическая волна, а материальная среда состоит из двухуровневых атомов. При этих условиях мы можем воспользоваться результатами, полученными ранее на базе флуктуационно-диссипационной теоремы в работе [17]. Обобщая эти результаты на случай с поперечным пространственным распределением поля, сможем 
написать для источников активной среды следующие корреляционные соотношения:

$$
\begin{gathered}
\left\langle\hat{F}_{i}^{\dagger}(\boldsymbol{\rho}, t) \hat{F}_{j}\left(\boldsymbol{\rho}^{\prime}, t^{\prime}\right)\right\rangle=\left\langle\hat{F}_{i} \hat{F}_{j}\right\rangle \delta\left(t-t^{\prime}\right) \delta^{2}\left(\boldsymbol{\rho}-\boldsymbol{\rho}^{\prime}\right), \\
\left\langle\hat{F}_{2} \hat{F}_{2}\right\rangle=\Gamma_{2} \bar{\sigma}_{2}+R_{a}\left(1-s_{a}\right), \\
\left\langle\hat{F}^{\dagger} \hat{F}\right\rangle=\Gamma_{1} \bar{\sigma}_{2}+R_{a}, \\
\left\langle\hat{F}_{1} \hat{F}_{1}\right\rangle=\Gamma_{1} \bar{\sigma}_{1} \\
\left\langle\hat{F}_{1} \hat{F}\right\rangle=\Gamma_{1} \bar{\sigma} \\
\left\langle\hat{F} \hat{F}_{2}\right\rangle=\Gamma_{2} \bar{\sigma} \\
\left\langle\hat{F} \hat{F}^{\dagger}\right\rangle=\Gamma_{2} \bar{\sigma}_{1}
\end{gathered}
$$

Здесь $\Gamma_{1}-$ скорость распада заселенности нижнего атомного уровня, $\Gamma_{2}-$ скорость распада заселенности верхнего атомного уровня, $R_{a}$ - скорость накачки верхнего атомного уровня, $s_{a}-$ статистический параметр накачки. Мы полагали, что скорость распада когерентности лазерного перехода $\Gamma=\left(\Gamma_{1}+\Gamma_{2}\right) / 2$. Здесь необходимо обратить внимание на параметр $0 \leq s_{a} \leq 1$, который дает возможность учитывать не только среднюю скорость возбуждения верхнего лазерного уровня $R_{a}$, но и следить за флуктуациями возбуждений. В предельном случае $s_{a}=0$ атомы возбуждаются независимо друг от друга и случайно во времени (пуассоновская накачка). В то же время при $s_{a}=1$ имеет место строго регулярная накачка без шумов (субпуассоновская накачка).

Используя те же самые подходы, которые развиты у [17], можем записать актуальные корреляционные функции для ланжевеновских источников пассивной среды в виде

$$
\begin{gathered}
\left\langle\hat{G}_{i}^{\dagger}(\boldsymbol{\rho}, t) \hat{G}_{j}\left(\boldsymbol{\rho}^{\prime}, t^{\prime}\right)\right\rangle=\left\langle\hat{G}_{i} \hat{G}_{j}\right\rangle \delta\left(t-t^{\prime}\right) \delta^{2}\left(\boldsymbol{\rho}-\boldsymbol{\rho}^{\prime}\right), \\
\left\langle\hat{G}_{1} \hat{G}_{1}\right\rangle=\gamma_{1} \bar{\pi}_{1}+R_{p}\left(1-s_{p}\right), \\
\left\langle\hat{G}^{\dagger} \hat{G}\right\rangle=\gamma_{1} \bar{\pi}_{2} \\
\left\langle\hat{G}_{2} \hat{G}_{2}\right\rangle=\gamma_{2} \bar{\pi}_{2} \\
\left\langle\hat{G} \hat{G}_{2}\right\rangle=\gamma_{2} \bar{\pi} \\
\left\langle\hat{G}_{1} \hat{G}\right\rangle=\gamma_{1} \bar{\pi} \\
\left\langle\hat{G} \hat{G}^{\dagger}\right\rangle=\gamma_{2} \bar{\pi}_{1}+R_{p} .
\end{gathered}
$$

Здесь $\gamma$ - скорость распада когерентности перехода с насыщенным поглощением, $\gamma_{1}-$ скорость распада заселенности нижнего атомного уровня, $\gamma_{2}-$ скорость распада заселенности верхнего атомного уровня, $R_{p}-$ скорость накачки нижнего атомного уровня, $s_{p}-$ статистический параметр накачки. Смысл параметра $s_{p}$ точно такой же, как $s_{a}$, но по отношению к нижнему уровню пассивной среды.

Корреляционные соотношения в $c$-числовом представлении для активной среды

$$
\begin{aligned}
& \left\langle F_{i}^{*}(\boldsymbol{\rho}, t) F_{j}\left(\boldsymbol{\rho}^{\prime}, t^{\prime}\right)\right\rangle \\
& =\left\langle F_{i}^{*}(\boldsymbol{\rho}, t) F_{j}(\boldsymbol{\rho}, t)\right\rangle \delta\left(t-t^{\prime}\right) \delta^{2}\left(\boldsymbol{\rho}-\boldsymbol{\rho}^{\prime}\right),
\end{aligned}
$$

$$
\begin{gathered}
\left\langle|F|^{2}\right\rangle=\Gamma_{1} \bar{\sigma}_{2}+R_{a}, \\
\left\langle F^{2}\right\rangle=2 g \overline{\sigma a}, \\
\left\langle F_{1} F\right\rangle=\Gamma_{1} \bar{\sigma}, \\
\left\langle F_{2} F_{2}\right\rangle=\Gamma_{2} \bar{\sigma}_{2}+R_{a}\left(1-s_{a}\right)-g\left(\overline{a^{*} \sigma}+\overline{a \sigma^{*}}\right), \\
\left\langle F_{1} F_{1}\right\rangle=\Gamma_{1} \bar{\sigma}_{1}-g\left(\overline{a^{*} \sigma}+\overline{a \sigma^{*}}\right), \\
\left\langle F_{2} F_{1}\right\rangle=g\left(\overline{a^{*} \sigma}+\overline{a \sigma^{*}}\right) .
\end{gathered}
$$

Корреляционные соотношения для пассивной среды

$$
\begin{gathered}
\left\langle G_{i}^{*}(\boldsymbol{\rho}, t) G_{j}\left(\boldsymbol{\rho}^{\prime}, t^{\prime}\right)\right\rangle=\left\langle G_{i}^{*} G_{j}\right\rangle \delta\left(t-t^{\prime}\right) \delta^{2}\left(\boldsymbol{\rho}-\boldsymbol{\rho}^{\prime}\right) \\
\left\langle|G|^{2}\right\rangle=R_{p}+\gamma_{1} \pi_{2}-\gamma_{1} \pi_{1}+\gamma_{2} \pi_{2}+2 g_{p}\left(\overline{a^{*} \pi}+\frac{(178)}{a \pi^{*}}\right), \\
\left\langle G^{2}\right\rangle=2 g_{p} \overline{\pi a}, \\
\left\langle G_{2} G\right\rangle=\gamma_{2} \bar{\pi} \\
\left\langle G_{1}^{2}\right\rangle=\gamma_{1} \bar{\pi}_{1}+R_{p}\left(1-s_{p}\right)-g_{p}\left(\overline{a * \pi}+\overline{a \pi^{*}}\right), \\
\left\langle G_{2}^{2}\right\rangle=\gamma_{2} \bar{\pi}_{2}-g_{p}\left(\overline{a^{*} \pi}+\overline{a \pi^{*}}\right), \\
\left\langle G_{1} G_{2}\right\rangle=g_{p}(182)
\end{gathered}
$$

\section{Список литературы}

[1] Розанов Н.Н. Диссипативные оптические солитоны. М.: Физматлит, 2011.

[2] Захаров В.Е., Манаков С.В., Новиков С.П., Питаевский Л.П. Теория солитонов: метод обратной задачи. М., Наука, 1980; Novikov S., Manakov S.V., Pitaevskii L.P., Zakharov V.E. Theory of Solitons. The Inverse Scattering Method. New York:, Consultants Bureau, 1984.

[3] Carter S.J., Drummond P.D., Reid M.D., Shelby R.M. // Phys. Rev. Lett. 1987. V. 58. P. 1841.

[4] Rosenbluh M., Shelby R.M. // Phys. Rev. Lett. 1991. V. 66. P. 153.

[5] Spalter S., Burk M., Strossner U., Sizmann A., Leuchs G. // Opt. Express. 1998. V. 2. P. 77.

[6] Mecozzi A., Kumar P. // Opt. Letters. 1997. V. 22. P. 1232.

[7] Lantz E., Sylvestre T., Maillotte H., Treps N., Fabre C. // J. Opt. B. Quantum Semiclass. Opt. 2004. V. 6. P. S295.

[8] Nagasako E.M., Boyd R.W., Agarwal G.S. // Opt. Express. 1998. V. 3. P. 171.

[9] Квантовое изображение / Под ред. М. Колобова. Физматлит, 2009; Oppo G.-L., Jeffers J. // Quantum Fluctuations in Cavity Solitons. Quantum Imaging. Ed. by Kolobov M.I. Berlin: Springer, 2007.

[10] Нестеров Л.А., Киселев Ал.С., Киселев Ан.С., Розанов Н.Н. // Опт. и спектр. 2009. Т. 106. № 4. С. 639-657; Nesterov L.A., Kiselev A.S., Kiselev A.S., Rosanov N.N. // Opt. Spectrosc. 2009. V. 106. P. 570.

[11] Нестеров Л.А., Веретенов Н.А., Розанов Н.Н. // Опт. и спектр. 2015. Т. 118. № 5. С. 815-827; Nesterov L.A., Veretenov N.A., Rosanov N.N. // Opt. Spectrosc. 2015. V. 118. P. 781-783.

[12] Нестеров Л.А., Веретенов Н.А., Розанов Н.Н. // Опт. и спектр. 2015. Т. 118. № 5. С. 828-836; Nesterov L.A., Veretenov N.A., Rosanov N.N. // Opt. Spectrosc. 2015. V. 118. P. 794. 
[13] Розанов Н.Н., Федоров С.В. // Опт. и спектр. 1992. Т. 72. № 6. C. 1394-1399; Rozanov N.N., Fedorov S.V. // Opt. Spectrosc. 1992. V. 72. P. 782.

[14] Sargent M.O., Scully M.O., Lamb W.E. Laser Physics, London: Addison-Wesley, 1974.

[15] Хакен Г., Вайдлих В. Квантовая теория лазера, М.: Мир, 1974. C. 143-205.

[16] Scully M.O., Zubairy M.S. Quantum Optics, Cambridge University Press, Cambridge, 1997.

[17] Davidovich L. // Rev. Mod. Phys. 1996. V. 68. P. 127.

[18] Golubev Y.M., Golubeva T.Y., Vashukevich E.A., Fedorov S.V., Rosanov N.N. // Laser Phys. Lett. 2019. V. 16. N 025201. 